\title{
An empirical formula for the mass-loss rates of dust-enshrouded red supergiants and oxygen-rich Asymptotic Giant Branch stars ${ }^{\star}$
}

\author{
J. Th. van Loon ${ }^{1}$, M.-R. L. Cioni ${ }^{2,3}$, A. A. Zijlstra ${ }^{4}$, and C. Loup ${ }^{5}$ \\ 1 Astrophysics Group, School of Physical \& Geographical Sciences, Keele University, Staffordshire ST5 5BG, UK \\ e-mail: jacco@astro.keele.ac.uk \\ 2 European Southern Observatory, Karl-Schwarzschild Straße 2, 85748 Garching bei München, Germany \\ 3 Institute for Astronomy, University of Edinburgh, Royal Observatory, Blackford Hill, Edinburgh EH9 3HJ, UK \\ 4 School of Physics and Astronomy, University of Manchester, Sackville Street, PO Box 88, Manchester M60 1QD, UK \\ ${ }^{5}$ Institut d'Astrophysique de Paris, 98bis boulevard Arago, 75014 Paris, France
}

Received 16 December 2004 / Accepted 12 April 2005

\begin{abstract}
We present an empirical determination of the mass-loss rate as a function of stellar luminosity and effective temperature, for oxygen-rich dust-enshrouded Asymptotic Giant Branch stars and red supergiants. To this aim we obtained optical spectra of a sample of dust-enshrouded red giants in the Large Magellanic Cloud, which we complemented with spectroscopic and infrared photometric data from the literature. Two of these turned out to be hot emission-line stars, of which one is a definite $\mathrm{B}[\mathrm{e}]$ star. The mass-loss rates were measured through modelling of the spectral energy distributions. We thus obtain the massloss rate formula $\log \dot{M}=-5.65+1.05 \log \left(L / 10000 L_{\odot}\right)-6.3 \log \left(T_{\text {eff }} / 3500 \mathrm{~K}\right)$, valid for dust-enshrouded red supergiants and oxygen-rich AGB stars. Despite the low metallicity of the LMC, both AGB stars and red supergiants are found at late spectral types. A comparison with galactic AGB stars and red supergiants shows excellent agreement between the mass-loss rate as predicted by our formula and that derived from the $60 \mu \mathrm{m}$ flux density for dust-enshrouded objects, but not for optically bright objects. We discuss the possible implications of this for the mass-loss mechanism.
\end{abstract}

Key words. stars: AGB and post-AGB - stars: carbon - stars: mass-loss - supergiants - Magellanic Clouds - infrared: stars

\section{Introduction}

Stellar death results in the injection of chemically enriched mass, energy and momentum into the interstellar medium. It also results in baryonic mass being locked up forever into degenerate objects (white dwarves, neutron stars and black holes). Together, these processes drive galactic chemical evolution and star formation.

Intermediate-mass stars, with Main-Sequence masses of $M_{\text {ZAMS }} \sim 1-8 M_{\odot}$, become Asymptotic Giant Branch (AGB) stars before ending as a white dwarf on timescales from only $t \simeq 30 \mathrm{Myr}$ up to as much as $10 \mathrm{Gyr}$. Luminous, $L_{\mathrm{AGB}} \sim 10^{4} L_{\odot}$, and cool, $T_{\text {eff,AGB }} \sim 2500-3500 \mathrm{~K}$, stars on the upper slopes of the AGB experience intense mass loss, losing up to 80 per cent of their original mass at rates of $\dot{M}_{\mathrm{AGB}} \sim 10^{-6}-10^{-4} M_{\odot} \mathrm{yr}^{-1}$ (Wood et al. 1983, 1992). Their luminosity is normally generated in a hydrogen-burning shell, isolated from the degenerate carbon-oxygen core and the overlying convective envelope, but episodically the temperature becomes sufficiently high to temporarily ignite a helium-burning shell (thermal pulses). This leads to penetration of the convective envelope, allowing for

* Based on observations obtained at the European Southern Observatory (La Silla, Chile). nuclear processed material to be transported to the stellar surface (e.g., carbon and s-process elements such as lithium and technetium). AGB stars within a certain mass range can thus be turned into carbon stars when the photospheric carbon-tooxygen abundance ratio exceeds unity (Bessell et al. 1983).

Massive stars also experience intense mass loss before they end through a supernova, leaving a neutron star or black hole (or deflagrating entirely) on timescales of $t \sim 1$ to $30 \mathrm{Myr}$. Of these the vast majority, stars with $M_{\text {ZAMS }} \sim 8-30 M_{\odot}$ pass through a red supergiant (RSG) stage, when they both are very luminous, $L_{\mathrm{RSG}} \sim 10^{5} L_{\odot}$, and become cool, $T_{\text {eff, RSG }} \sim$ 3500-4000 K (Massey \& Olsen 2003). As red supergiants they loose mass at rates of $\dot{M} \sim 10^{-5}-10^{-3} M_{\odot} \mathrm{yr}^{-1}$, but there is a lot of uncertainty about the total amount of mass lost during the RSG phase, and therefore about the exact nature of the immediate supernova progenitors (Filippenko 1997).

AGB and RSG mass loss is essentially driven by the same mechanism: radiation pressure on dust grains which condense in the elevated atmospheres of radially pulsating giant stars and drag the gas along with it through mutual collisions. It seems therefore logical that the mass-loss rate would depend on the stellar luminosity (radiation pressure) and temperature (to allow dust to form). Indeed, both theory and observations 
confirm that this is the case. However, even the most sophisticated, hydrodynamical models suffer from a range of assumptions and are so far only presented for carbon stars, at an initial solar metallicity (e.g., Wachter et al. 2002; Sandin \& Höfner 2003). Mass-loss rates have been measured for a sample of AGB stars and red supergiants in the Large Magellanic Cloud (LMC) by van Loon et al. (1999b), who demonstrate that the mass-loss rate increases with increasing luminosity, and for increasingly late spectral type for oxygen-rich (M-type) AGB stars and red supergiants, but they do not quantify these dependencies.

We here present a recipe for the mass-loss rate as a function of luminosity and stellar effective temperature, $\dot{M}\left(L, T_{\text {eff }}\right)$, for oxygen-rich AGB stars and red supergiants in the LMC, which undergo strong regular pulsations (Whitelock et al. 2003) and are enshrouded in dusty envelopes. This is the first empirical relationship of its kind, that can be readily applied in models for stellar and galactic evolution. Although much work has been done on the mass-loss rates of cool giants in globular clusters and in the solar vicinity (e.g., Reimers 1975a,b; Jura \& Kleinmann 1992; Catelan 2000), these studies concentrate on first ascent RGB stars and low-mass AGB stars with moderate mass-loss rates. Our formula is the first attempt to quantify the relationship between the measured values for the mass-loss rate, bolometric luminosity and stellar effective temperature for dusty cool giants. Valid well into the superwind regime of extremely high mass-loss rates, our formula provides a more direct alternative to the commonly used extra-polations of relationships which were derived for less massive and less evolved red giants.

As part of this investigation, we obtained optical spectra for a number of highly obscured objects for which no such information was available, to estimate their stellar effective temperatures. We compiled infrared (IR) photometry to sample the spectral energy distributions which were then modelled with a dust radiative transfer code in order to derive the mass-loss rates. We also present a more qualitative analysis of a small sample of carbon stars and (M)S-type stars with a carbon-tooxygen ratio slightly less than unity, as well as a comparison with galactic AGB stars and red supergiants.

\section{Observational data}

\subsection{Spectroscopy}

Spectra of (candidate) dust-enshrouded AGB stars and red supergiants were obtained on three occasions $(1995,1998$ and 2000). DFOSC at the $1.5 \mathrm{~m}$ Danish telescope at La Silla, Chile, was used from 8 to 11 December 1995 to obtain low-resolution $(R \sim 900)$ long-slit spectra between $\sim 0.41$ and $0.68 \mu \mathrm{m}$ with grism \#7 and a $1.5^{\prime \prime}$ slit width. EMMI at the ESO $3.5 \mathrm{~m}$ NTT at La Silla, Chile, was used on 24 December 1998 and on 15 and 16 January 2000, to obtain low-resolution $(R \sim 500)$ long-slit spectra between $\sim 0.61$ and $1.04 \mu \mathrm{m}$ with grism \#4 and a $1.5^{\prime \prime}$ slit width. Integration times ranged between 5 and 30 min.

The data were reduced using standard procedures in ESO-Midas. The frames were corrected for the electronic offset
Table 1. Spectroscopic targets, listed in order of increasing Right Ascension (J2000 coordinates). The last column refers to the observing run, where "D5" = DFOSC 1995, "E8" = EMMI 1998 and "E0" = EMMI 2000.

\begin{tabular}{lccc}
\hline \hline Object & RA & Dec & Run \\
\hline IRAS 04407-7000 & 44028.5 & -695514 & E8 \\
IRAS 04498-6842 & 44941.5 & -683752 & E8 \\
IRAS 04530-6916 & 45245.7 & -691150 & E8 \\
WOH G64 & 45510.5 & -682030 & D5 \\
IRAS 05003-6712 & 50019.0 & -670758 & E8 \\
SP77 28-10 & 50023.0 & -655959 & E0 \\
DCMC J050024.95-710130.7 & 50025.0 & -710131 & E0 \\
SHV 0500533-690617 & 50038.2 & -690201 & E0 \\
SHV 0504018-690157 & 50346.8 & -685754 & E0 \\
SHV 0504353-712622 & 50356.0 & -712222 & E0 \\
SHV 0504266-691145 & 50410.1 & -690743 & E0 \\
DCMC J050615.14-720657.1 & 50615.1 & -720657 & E0 \\
DCMC J050659.94-634746.7 & 50659.9 & -634747 & E0 \\
GRV 0507-6616 & 50710.3 & -661243 & E0 \\
DCMC J050738.74-733233.4 & 50738.7 & -733233 & E0 \\
IRAS 05128-6455 & 51304.6 & -645140 & E8 \\
HV 916 & 51449.7 & -672720 & D5 \\
GRV 0519-6700 & 51942.0 & -665749 & D5 \\
HV 2561 & 52828.9 & -680708 & D5 \\
IRAS 05294-7104 & 52848.2 & -710229 & E8 \\
IRAS 05289-6617 & 52902.4 & -661528 & E8 \\
SP77 46-44 & 52942.2 & -685717 & D5 \\
HV 986 & 53109.3 & -672555 & D5 \\
LI-LMC 1522 & 54013.3 & -692247 & E8 \\
\hline & & &
\end{tabular}

(bias) and the relative pixel response (flatfield). Wavelength calibration was performed relative to $\mathrm{He}+\mathrm{Ar}$ or $\mathrm{He}+\mathrm{Ne}$ lamp spectra. The sky-subtracted spectra were corrected for the wavelength dependence of the instrumental response as measured from the spectrum of a standard star, and for atmospheric continuum extinction.

The targets (Table 1) were chosen from samples of mid-IR sources with known optically visible counterparts (dominating the DFOSC 1995 run) - mainly from Loup et al. (1997), bright mid-IR sources associated with heavily dust-enshrouded objects (mostly during the EMMI 1998 run) - cf. Zijlstra et al. (1996), and red objects that were likely to be carbon stars (EMMI 2000 run). The latter were selected from Cioni et al. (2000) to add to the previous samples that turned out to be predominantly oxygen-rich. Several galactic $\mathrm{M}$ giants were also observed for reference (see Appendix A). 
Table 2. Spectral classification and near-IR magnitudes (wavelengths in $\mu \mathrm{m}$ ).

\begin{tabular}{|c|c|c|c|c|c|c|c|c|}
\hline \multirow[t]{2}{*}{ Object } & \multicolumn{2}{|c|}{ Spectral type } & \multicolumn{3}{|c|}{ DENIS } & \multicolumn{3}{|c|}{ 2MASS } \\
\hline & Here & Previous & 0.79 & 1.23 & 2.15 & 1.24 & 1.66 & 2.16 \\
\hline \multicolumn{9}{|l|}{ Stars of type $M$} \\
\hline HV 12501 & & M 1.5 & 10.08 & 8.62 & 7.51 & 8.80 & 8.03 & 7.70 \\
\hline HV 2360 & & M 2 Ia & 10.53 & & 7.45 & 8.78 & 7.98 & 7.59 \\
\hline HV 2446 & & M 5e & 14.38 & 10.56 & 8.97 & 10.67 & 9.81 & 9.36 \\
\hline HV 2561 & M 1.5 & M OIa & 10.09 & 8.26 & 6.79 & 8.61 & 7.80 & 7.48 \\
\hline HV 5870 & & $\mathrm{M} 4.5 / 5$ & 10.95 & & 7.70 & 9.10 & 8.26 & 7.90 \\
\hline HV 888 & & $\mathrm{M} 4 \mathrm{Ia}$ & 9.66 & 7.88 & & 8.01 & 7.19 & 6.78 \\
\hline HV 916 & M 2.5 & M3Iab & 10.47 & & 7.34 & 8.64 & 7.78 & 7.42 \\
\hline HV 986 & M2 & & & & & 8.80 & 7.95 & 7.63 \\
\hline HV 996 & & M 4Iab & 10.71 & 8.82 & & 8.99 & 8.16 & 7.64 \\
\hline IRAS $04407-7000$ & M7.5 & & 13.73 & 10.09 & 8.11 & 10.66 & 9.49 & 8.69 \\
\hline IRAS 04498-6842 & M 10 & & 14.96 & 10.67 & 8.40 & 9.13 & 8.05 & 7.49 \\
\hline IRAS 04509-6922 & & M 10 & & & & 9.87 & 8.67 & 7.93 \\
\hline IRAS 04516-6902 & & M9 & 17.39 & 11.35 & & 9.93 & 8.62 & 7.91 \\
\hline IRAS 05003-6712 & M9 & & 18.29 & 13.17 & 10.32 & 12.04 & 10.46 & 9.32 \\
\hline IRAS $05128-6455$ & M9 & & 17.87 & 13.43 & & 14.55 & 12.83 & 11.28 \\
\hline IRAS 05294-7104 & M 8 & & & 12.87 & 9.67 & 12.99 & 11.19 & 9.88 \\
\hline SHV 0522023-701242 & & M3 & 16.67 & 13.29 & & 12.63 & 11.74 & 11.31 \\
\hline SHV 0530323-702216 & & M 6 & 14.66 & 11.49 & 9.86 & 11.79 & 10.89 & 10.39 \\
\hline SP77 30-6 & & M 8 & 14.61 & 11.06 & 9.36 & 11.14 & 10.28 & 9.55 \\
\hline SP77 46-44 & M 1.5 & M 1 Ia & 9.53 & 7.83 & 6.38 & 7.92 & 7.19 & 6.89 \\
\hline WOH G64 & $\mathrm{M} 7.5 \mathrm{e}$ & M7.5 & 13.04 & 9.44 & & 9.25 & 7.74 & 6.85 \\
\hline WOH SG374 & & M 6 & & & & 9.80 & 9.18 & 8.67 \\
\hline \multicolumn{9}{|l|}{ Stars of type $M S$ and $S$} \\
\hline GRV 0519-6700 & S5,2 & & 14.81 & 13.04 & 10.83 & 12.17 & 11.17 & 10.59 \\
\hline HV 12070 & & MS3/9 & 14.37 & 10.43 & 9.02 & 10.61 & 9.75 & 9.20 \\
\hline SHV 0524565-694559 & & MS5 & 14.26 & 12.06 & 10.24 & 11.60 & 10.67 & 10.27 \\
\hline \multicolumn{9}{|l|}{ Stars of type $C$} \\
\hline DCMC J050024.95-710130.7 & $\mathrm{C} 9,5+$ & & 15.31 & 13.02 & 10.33 & 12.46 & 11.17 & 10.10 \\
\hline DCMC J050615.14-720657.1 & $\mathrm{C} 6,3$ & & 15.18 & 13.06 & 10.25 & 13.13 & 11.64 & 10.35 \\
\hline DCMC J050659.94-634746.7 & $\mathrm{C} 9,5$ & & 14.35 & 12.35 & 10.12 & 12.45 & 11.08 & 10.14 \\
\hline DCMC J050738.74-733233.4 & $\mathrm{C} 1, ?$ & & 14.45 & 12.17 & 9.73 & 13.12 & 11.60 & 10.43 \\
\hline GRV 0507-6616 & $\mathrm{C} 8,4$ & & 15.45 & 13.35 & 10.62 & 12.99 & 11.53 & 10.50 \\
\hline IRAS 05289-6617 & $\mathrm{C} 10,1$ & & 15.99 & 14.32 & 12.77 & 14.77 & 13.62 & 13.02 \\
\hline SHV 0500533-690617 & C 8,5+ & & 16.12 & 13.54 & 11.13 & 13.92 & 12.39 & 11.44 \\
\hline SHV 0504018-690157 & $\mathrm{C} 5,4$ & & 15.78 & 13.53 & 10.54 & 13.59 & 11.94 & 10.69 \\
\hline SHV 0504266-691145 & $\mathrm{C} 8,4$ & & 15.23 & 13.05 & 10.67 & 14.38 & 12.72 & 11.47 \\
\hline SHV 0504353-712622 & $\mathrm{C} 7,5$ & & 14.87 & 12.78 & 10.48 & 12.37 & 11.13 & 10.41 \\
\hline SP77 28-10 & $\mathrm{C} 9,3$ & & 15.85 & 13.48 & 10.71 & 12.99 & 11.56 & 10.51 \\
\hline \multicolumn{9}{|l|}{ Emission-line stars } \\
\hline IRAS 04530-6916 & em. & & 15.72 & 13.47 & 9.61 & 13.94 & 11.86 & 9.96 \\
\hline LI-LMC 1522 & em. & & 10.93 & 10.31 & 8.43 & 10.46 & 9.85 & 8.60 \\
\hline
\end{tabular}

\subsection{Infrared photometry}

In order to estimate bolometric luminosities and mass-loss rates, infrared photometry was compiled to sample the spectral energy distribution (SED). Near-IR photometry (Table 2) was retrieved from the DENIS catalogue (IJK $\mathrm{s}$ bands at $0.79,1.23$ and $2.15 \mu \mathrm{m}$; Cioni et al. 2000; cf. Fouqué et al. 2000) and the 2MASS catalogue $\left(J H K_{\mathrm{s}}\right.$ bands at $1.24,1.66$ and $2.16 \mu \mathrm{m}$; cf. Cohen et al. 2003). Mid-IR photometry (Table 3) was retrieved from the MSX catalogue or from the original MSX images, 
Table 3. Mid-IR flux densities (in Jy, with the wavelengths in $\mu \mathrm{m}$ ). The column "s" indicates the availability of an ISO spectrum ("y"). Values accompanied by a colon are uncertain, whilst question marks indicate detections that are suspect.

\begin{tabular}{|c|c|c|c|c|c|c|c|c|c|c|c|}
\hline \multirow[t]{2}{*}{ Object } & \multicolumn{4}{|c|}{ MSX } & \multicolumn{3}{|c|}{ ISO } & \multicolumn{4}{|c|}{ IRAS } \\
\hline & 8.28 & 12.13 & 14.65 & 21.34 & 12 & 25 & $\mathrm{~s}$ & 12 & 25 & 60 & 100 \\
\hline \multicolumn{12}{|l|}{ Stars of type $M$} \\
\hline HV 12501 & 0.108 & $<0.05$ & $<0.08$ & $<0.4$ & 0.19 & 0.07 & & 0.20 & 0.08 & $<0.2$ & \\
\hline HV 2360 & 0.321 & 0.41 & 0.25 & $<0.8$ & 0.33 & 0.14 & & 0.38 & 0.35 & 0.4: & \\
\hline HV 2446 & 0.033: & & & & 0.07 & 0.04 & $\mathrm{y}$ & 0.05 & 0.02 & $<0.2$ & \\
\hline HV 2561 & 0.245 & $0.18:$ & $0.15:$ & 0.5 : & & & & 0.28 & 0.22 & $<1.0$ & \\
\hline HV 5870 & 0.144 & $<0.12$ & $<0.06$ & $<0.5$ & 0.27 & 0.09 & & 0.30 & 0.17 & $<5.0$ & \\
\hline HV 888 & 0.430 & 0.51 & 0.17 & $<0.2$ & 0.71 & 0.20 & $\mathrm{y}$ & 0.58 & 0.29 & $<4.0$ & \\
\hline HV 916 & 0.267 & $0.23:$ & 0.27 : & $<0.4$ & 0.38 & 0.18 & & 0.44 & 0.23 & $<2.0$ & \\
\hline HV 986 & 0.076 & $<0.19$ & $<0.13$ & $<0.5$ & & & & 0.11 & 0.06: & $<0.6$ & \\
\hline HV 996 & 0.457 & 0.67 & 0.27 & 0.3 : & 0.60 & 0.36 & $\mathrm{y}$ & 0.71 & 0.53 & $<0.5$ & \\
\hline IRAS 04407-7000 & 0.424 & 0.46 & 0.51 & 0.7 & 0.96 & 0.58 & & 0.76 & 0.76 & 0.1 & \\
\hline IRAS $04498-6842$ & 0.321 & 0.64 & 0.73 & $<0.5$ & 0.49 & 0.22 & & 1.33 & 0.89 & $<0.2$ & \\
\hline IRAS 04509-6922 & 0.181 & $<0.20$ & $0.34:$ & $<0.3$ & 0.30 & 0.20 & & 0.89 & 0.86 & $<2.0$ & \\
\hline IRAS 04516-6902 & 0.318 & 0.21 & $<0.18$ & $<0.4$ & 0.85 & 0.38 & & 0.86 & 0.55 & 0.4: & \\
\hline IRAS 05003-6712 & 0.155 & $0.34:$ & $0.17:$ & $<0.1$ & 0.36 & 0.21 & $\mathrm{y}$ & 0.43 & 0.33 & $0.1:$ & \\
\hline IRAS 05128-6455 & 0.196 & $<0.28$ & $0.22:$ & $<0.2$ & 0.23 & 0.06 & $\mathrm{y}$ & 0.23 & 0.24 & 0.1 & \\
\hline IRAS 05294-7104 & 0.177 & $0.41:$ & $<0.22$ & $<0.4$ & 0.68 & 0.39 & & 0.69 & 0.56 & $<3.0$ & \\
\hline SHV 0522023-701242 & $<0.010$ & & & & 0.001: & $<0.02$ & & $<0.10$ & $<0.04$ & $0.4:$ & \\
\hline SHV $0530323-702216$ & 0.011: & & & & 0.008 & 0.008 : & & $<0.04$ & $<0.04$ & 0.4 & \\
\hline SP77 30-6 & 0.089 & $<0.12$ & $<0.15$ & $<0.5$ & 0.14 & 0.08 & $\mathrm{y}$ & 0.26 & 0.13 & $0.1:$ & \\
\hline SP77 46-44 & 0.192 & $0.17:$ & $<0.21$ & $<0.8$ & & & & 0.26 & 0.18 & $<0.2$ & \\
\hline WOH G64 & 6.220 & 8.73 & 9.71 & 11.6 & 12.11 & 13.79 & $\mathrm{y}$ & 8.45 & 13.53 & 2.2 & $<16.0$ \\
\hline WOH SG374 & 0.254 & 0.44 & $0.39:$ & $<0.2$ & 0.49 & 0.19 & & 0.37 & 0.38 & $0.2:$ & \\
\hline \multicolumn{12}{|l|}{ Stars of type $M S$ and $S$} \\
\hline GRV 0519-6700 & $<0.023$ & & & & 0.004 & & & $<0.06$ & $<0.03$ & 0.08 : & \\
\hline HV 12070 & 0.054 & & & & 0.043 & $<0.02$ & $\mathrm{y}$ & 0.06 & 0.03 & $0.1:$ & \\
\hline SHV 0524565-694559 & $<0.008$ & & & & 0.003 & & & $<0.14$ & $<0.07$ & $<1.0$ & \\
\hline \multicolumn{12}{|l|}{ Stars of type $C$} \\
\hline DCMC J050024.95-710130.7 & 0.074 & & & & & & & $0.07 ?$ & $0.05 ?$ & $<0.1$ & \\
\hline DCMC J050615.14-720657.1 & $0.022:$ & & & & & & & 0.10 & 0.04 & $<0.2$ & \\
\hline DCMC J050659.94-634746.7 & $<0.020$ & & & & & & & $<0.10$ & $<0.10$ & $<0.2$ & \\
\hline DCMC J050738.74-733233.4 & 0.041 & & & & & & & 0.07 & $0.02:$ & $<0.2$ & \\
\hline GRV 0507-6616 & 0.022: & & & & & & & $<0.04$ & 0.05: & $<0.2$ & \\
\hline IRAS 05289-6617 & 0.064 & $<0.41$ & $0.10:$ & 0.2 : & 0.16 & 0.20 & $\mathrm{y}$ & 0.16 & 0.39 & 0.3 & \\
\hline SHV 0500533-690617 & 0.034: & & & & & & & $0.05 ?$ & $<0.08$ & $<0.6$ & \\
\hline SHV 0504018-690157 & 0.045: & & & & & & & 0.03 & $<0.13$ & $<0.6$ & \\
\hline SHV 0504266-691145 & 0.029: & & & & & & & $0.02:$ & $<0.10$ & $<2.0$ & \\
\hline SHV 0504353-712622 & $<0.009$ & & & & & & & $0.02 ?$ & $0.03 ?$ & $0.5 ?$ & \\
\hline SP77 28-10 & 0.031 & & & & & & & $<0.15$ & $<0.11$ & $<0.9$ & \\
\hline \multicolumn{12}{|l|}{ Emission-line stars } \\
\hline IRAS 04530-6916 & 1.144 & 1.93 & 1.72 & 2.5 & & & & 2.07 & 5.09 & 22.0 & 28.0 \\
\hline LI-LMC 1522 & 0.868 & 0.45 & 1.05 & 1.2 & & & & 1.00 & 0.92 & $<3.0$ & \\
\hline
\end{tabular}


from ISO (Trams et al. 1999), and from IRAS scans following the procedure described in Trams et al. (1999). Upper limits were determined where these would provide useful constraints on the shape of the SED, noting that the MSX upper limits correspond to $1-\sigma$ levels only.

\subsection{Additional samples}

We complement our sample with objects from van Loon et al. (1998) and Trams et al. (1999) for which spectral subclassification and mid-IR photometry is available. No more carbon stars were included in this way, but several M-type and two MS-type stars were added. The MS star SHV 0524565-694559 was detected in the ISO mini-survey of the LMC (Loup et al., in preparation), with $F_{4.5}=16.2 \pm 2.3 \mathrm{mJy}$ and $F_{12}=$ $5.3 \pm 0.7 \mathrm{mJy}$. Another possible $\mathrm{S}$ star listed in Trams et al. is SHV 0522118-702517, but its classification is uncertain and no subclassification is given.

A further 34 IRAS-detected objects with spectral subclassification are available from Loup et al. (1997; their Table 1); these are predominantly early-M type supergiants that are already well represented in our sample.

\section{Analysis}

\subsection{Spectral classification}

The spectral type of oxygen-rich (M-type) stars was derived from the relative strengths of the $\mathrm{TiO}$ and $\mathrm{VO}$ molecular absorption bands, and of the Ca II triplet, whilst the spectral type of carbon-rich (C-type) stars was derived from the relative strengths of the $\mathrm{C}_{2}$ and $\mathrm{CN}$ molecular absorption bands. S-type stars with a carbon abundance approaching that of oxygen have prominent $\mathrm{ZrO}$ bands, with MS-type stars representing a milder form of this chemical peculiarity. For more details about these bands the reader is referred to Turnshek et al. (1985).

\subsubsection{M-type stars}

The M-type stars observed with DFOSC (Fig. 1) turn out to be red supergiants, whilst the M-type stars observed with EMMI (Fig. 2) are on the AGB (see Table 4, Fig. 12). The 4000 to $7000 \AA$ region is not optimal for classification of late-M types. The 7000 to $9000 \AA$ region is much better, chiefly due to the diagnostic value of the VO bands. There can be some ambiguity, notably in WOH G64 (Fig. 1) where various band strengths (in particular at $6200 \AA$ ) suggest a very late type (M 7-8) whilst some relative band strengths (e.g., 6200 compared to $5900 \AA$ ) suggest a much earlier type ( M 5). Band strength ratios might better reflect the excitation temperature, whilst individual bands may appear stronger as a result of enhanced optical depth in the puffed up atmospheres of masslosing giants. Nonetheless, normally we obtain a spectral classification that is accurate enough for our purposes.

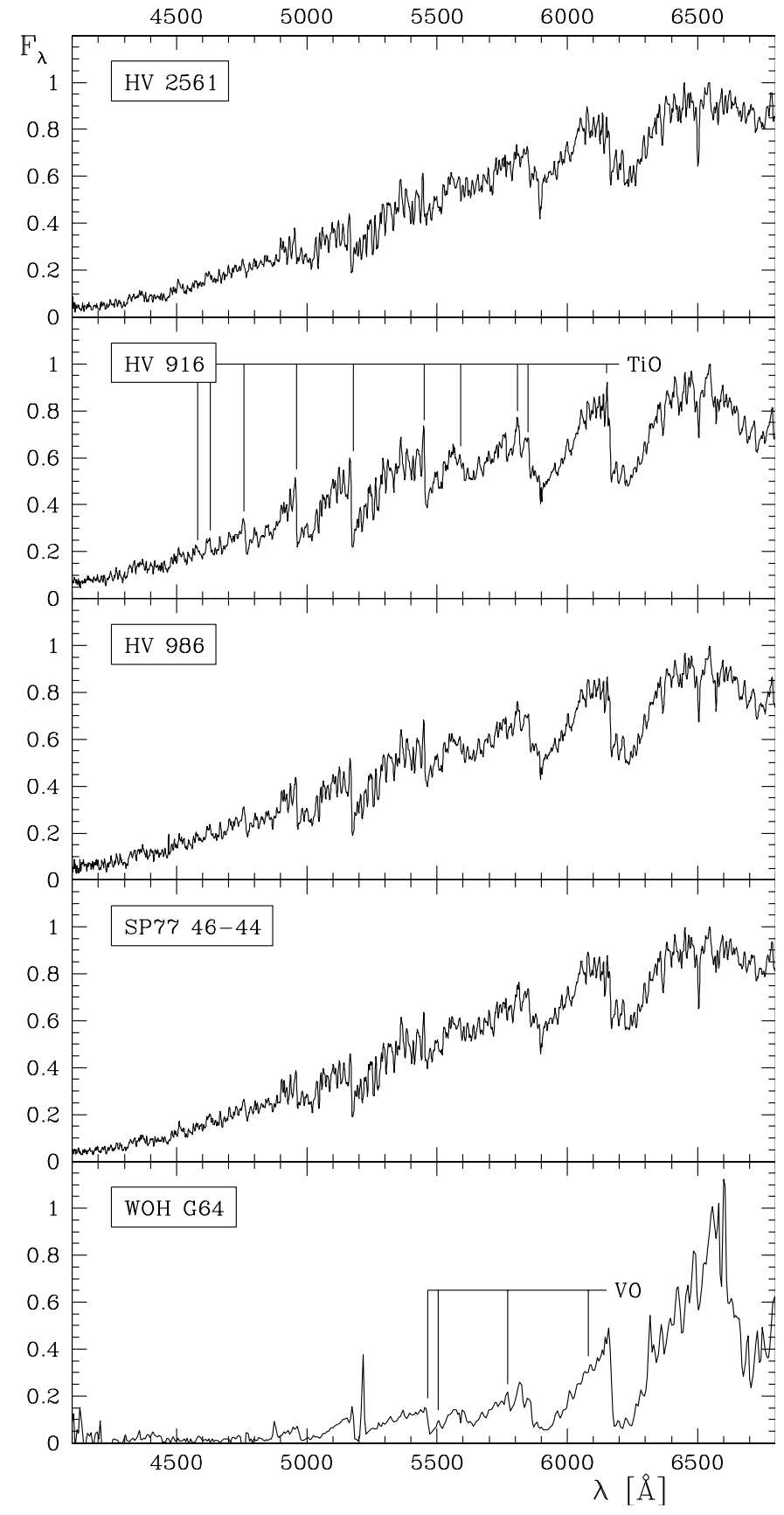

Fig. 1. DFOSC spectra of M-type supergiants.

\subsubsection{An S-type star}

In S-type stars, the $5469 \AA$ VO band becomes weaker (e.g., relative to the $\mathrm{ZrO}$ bands) with increasing $\mathrm{C} / \mathrm{O}$ abundance ratio. It is quite strong in GRV 0519-6700 (Fig. 3), which must therefore have a $\mathrm{C} / \mathrm{O}$ ratio not too close to unity. The YO bands at 5972 and $6132 \AA$, and the pair of roughly symmetrical $\mathrm{CaCl}$ bands around $6200 \AA$ only appear in the cooler S-type stars (Keenan \& Boeshaar 1980), and they are clearly present in the spectrum of GRV 0519-6700. There is no sign of ${ }^{13} \mathrm{CN}$ bands suppressing the peak in the spectrum between 6450 and $6456 \AA$, and one can assume that this star is not particularly ${ }^{13} \mathrm{C}$ rich. 


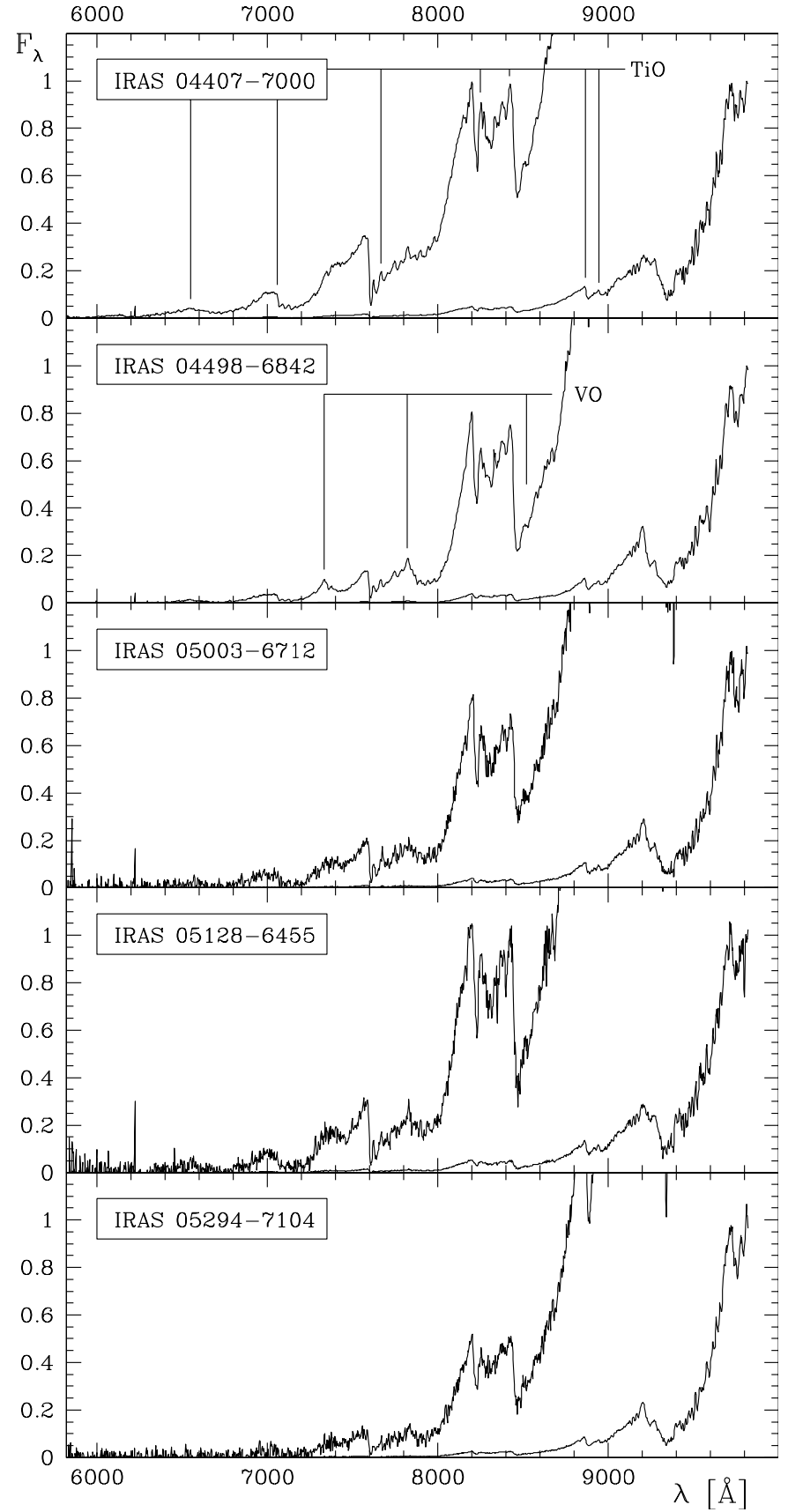

Fig. 2. EMMI spectra of M-type AGB stars, displayed twice on different scales to exploit the full dynamic range of the data.

\subsubsection{Carbon stars}

The spectra of carbon stars (Figs. 4 and 5) are generally quite similar, with a few exceptions. The $\mathrm{C} / \mathrm{O}$ abundance ratio determines the relative strength of the $\mathrm{C}_{2}$ and $\mathrm{CN}$ bands. The $\mathrm{C}_{2}$ bands are best measured shortward of $5600 \AA$, but unfortunately that spectral region was not included (and the stars are much fainter in the $V$ band). The best diagnostic in our spectral coverage is the collection of $\mathrm{C}_{2}$ bands shortward of $6200 \AA$, and around 7700 and $8800 \AA$. The temperature is best measured from the strength of the $\mathrm{CN}$ bands, roughly between 6300 and $7500 \AA$.
Table 4. Results from the modelling of the spectral energy distributions: the stellar effective temperature (from the optical spectrum), $T_{\text {eff }}$, the temperature at the inner radius of the dust envelope, $T_{\text {dust }}$, the wind speed, $v$, the bolometric luminosity, $L$, and the total (gas+dust) mass-loss rate, $\dot{M}$. We omitted the DCMC prefix to the first four carbon stars.

\begin{tabular}{|c|c|c|c|c|c|}
\hline Object & $\begin{array}{c}T_{\text {eff }} \\
\mathrm{K} \\
\end{array}$ & $\begin{array}{c}T_{\text {dust }} \\
\mathrm{K} \\
\end{array}$ & $\begin{array}{c}v \\
\mathrm{~km} \mathrm{~s}^{-1}\end{array}$ & $\begin{array}{c}\log L \\
L_{\odot} \\
\end{array}$ & $\begin{array}{c}\log \dot{M} \\
M_{\odot} / \mathrm{yr}\end{array}$ \\
\hline \multicolumn{6}{|l|}{ Stars of type $M$} \\
\hline HV 12501 & 3773 & 800 & 17.5 & 5.16 & -4.79 \\
\hline HV 2360 & 3736 & 700 & 15.8 & 5.11 & -4.51 \\
\hline HV 2446 & 3434 & 600 & 7.8 & 4.35 & -5.14 \\
\hline HV 2561 & 3831 & 450 & 11.9 & 5.25 & -4.47 \\
\hline HV 5870 & 3469 & 700 & 12.7 & 4.98 & -4.68 \\
\hline HV 888 & 3574 & 800 & 19.7 & 5.43 & -4.43 \\
\hline HV 916 & 3684 & 600 & 14.5 & 5.17 & -4.50 \\
\hline HV 986 & 3736 & 450 & 9.7 & 5.12 & -4.89 \\
\hline HV 996 & 3574 & 600 & 12.3 & 5.07 & -4.28 \\
\hline IRAS 04407-7000 & 3008 & 900 & 10.9 & 4.74 & -4.15 \\
\hline IRAS $04498-6842$ & 2500 & 1100 & 14.4 & 4.89 & -4.30 \\
\hline IRAS 04509-6922 & 2500 & 800 & 11.7 & 4.83 & -4.45 \\
\hline IRAS 04516-6902 & 2667 & 900 & 12.6 & 4.89 & -4.23 \\
\hline IRAS 05003-6712 & 2667 & 1200 & 10.5 & 4.39 & -4.34 \\
\hline IRAS 05128-6455 & 2667 & 1000 & 7.2 & 4.08 & -4.36 \\
\hline IRAS 05294-7104 & 2890 & 900 & 7.9 & 4.42 & -4.14 \\
\hline SHV 0522023-701242 & 3666 & 1200 & 6.9 & 3.56 & -6.80 \\
\hline SHV 0530323-702216 & 3309 & 500 & 4.5 & 3.88 & -5.67 \\
\hline SP77 30-6 & 2890 & 800 & 8.5 & 4.29 & -4.76 \\
\hline SP77 46-44 & 3792 & 400 & 10.9 & 5.47 & -4.56 \\
\hline WOH G64 & 3008 & 800 & 15.8 & 5.69 & -3.12 \\
\hline WOH SG374 & 3309 & 600 & 8.4 & 4.68 & -4.43 \\
\hline \multicolumn{6}{|l|}{ Stars of type $M S$ and $S$} \\
\hline GRV 0519-6700 & 3300 & 1200 & 8.8 & 3.70 & -6.20 \\
\hline HV 12070 & 3600 & 750 & 10.8 & 4.41 & -5.20 \\
\hline SHV 0524565-694559 & 3400 & 1200 & 7.7 & 3.90 & -6.53 \\
\hline \multicolumn{6}{|l|}{ Stars of type $C$} \\
\hline J050024.95-710130.7 & 2380 & 800 & 8.9 & 4.09 & -5.05 \\
\hline J050615.14-720657.1 & 2920 & 1000 & 9.8 & 4.03 & -4.96 \\
\hline J050659.94-634746.7 & 2380 & 1300 & 19.5 & 3.92 & -5.64 \\
\hline J050738.74-733233.4 & 3820 & 1200 & 14.9 & 4.05 & -5.07 \\
\hline GRV 0507-6616 & 2560 & 1300 & 17.6 & 3.77 & -5.59 \\
\hline IRAS 05289-6617 & 2200 & 220 & 1.5 & 3.69 & -4.35 \\
\hline SHV 0500533-690617 & 2560 & 900 & 8.6 & 3.63 & -5.39 \\
\hline SHV 0504018-690157 & 3100 & 1200 & 13.3 & 3.79 & -5.30 \\
\hline SHV 0504266-691145 & 2560 & 1000 & 10.3 & 3.62 & -5.48 \\
\hline SHV 0504353-712622 & 2740 & 1200 & 15.7 & 3.91 & -5.62 \\
\hline SP77 28-10 & 2380 & 1100 & 13.9 & 3.80 & -5.51 \\
\hline
\end{tabular}




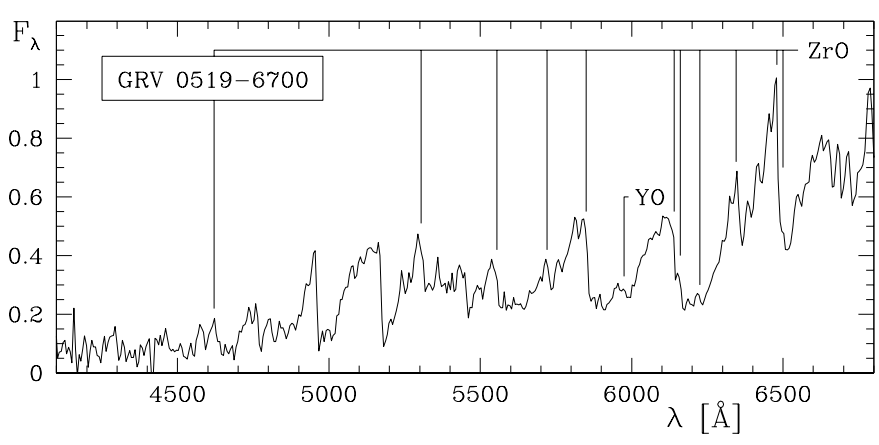

Fig. 3. DFOSC spectrum of the S-type star GRV 0519-6700.

DCMC 050738.74-733233.4 (Fig. 4) seems to be a warm carbon star and may either be extrinsic (i.e., enriched by material from a previous AGB companion), experiencing the effects of a thermal pulse, or on a post-AGB track. As a consequence of the relatively high temperature all bands become weak and it is therefore impossible to estimate the $\mathrm{C} / \mathrm{O}$ ratio from our spectrum.

IRAS 05289-6617 (Fig. 5) exhibits extremely strong absorption in some of the $\mathrm{CN}$ bands, with no sign of $\mathrm{C}_{2}$. This dust-enshrouded object might be nitrogen-enriched, possibly as a result of (mild) HBB. The mid-IR ISOCAM-CVF spectrum (Trams et al. 1999) shows an emission feature around $11.5 \mu \mathrm{m}$ that could either be due to $\mathrm{SiC}$ grains or Polycyclic Aromatic Hydrocarbons (PAHs). This star is located at the fringes of the populous elliptical intermediate-age cluster NGC 1978 and its colours suggest the lack of warm dust (van Loon et al., in preparation). The latter might be explained if the star has just recovered from a thermal pulse.

\subsubsection{Emission-line objects}

Two dusty objects turned out to exhibit a rich spectrum of strong emission lines (Fig. 6) including Balmer $\mathrm{H} \alpha$, the $\mathrm{Ca}$ II triplet and the Paschen hydrogen series longward of $8400 \AA$. There is evidence for a two-component spectrum, with a warm (or hot) continuum peaking around $6000 \AA$ and a cold continuum dominating longward of $9000 \AA$. The cool component seems to be relatively more important in IRAS 04530-6916 than in LI-LMC 1522.

IRAS 04530-6916 has strong [S II] emission in the $6717+6731 \AA$ doublet. This very luminous object was interpreted as a candidate massive Young Stellar Object on the basis of its IR colours (van Loon et al. 2001b).

Besides the broad $\mathrm{H} \alpha$ line, LI-LMC 1522 shows strong He I emission lines at 6678 and $7065 \AA$ as well as emission in [Ca II] at 7291+7324 $\AA$. Strong He lines are also seen in objects such as $\eta$ Carinae (Wallerstein et al. 2001), and LI-LMC 1522 may be an evolved supergiant. Indeed, its spectrum resembles that of the $\mathrm{B}[\mathrm{e}]$ supergiant Hen $\mathrm{S} 134$ and like that luminous B0 star also shows the $6170 \AA \mathrm{TiO}$ band in emission and numerous Fe II emission lines in the 6000-6500 $\AA$ region (Lamers et al. 1998).

When taking the spectrum of the dust-enshrouded AGB star IRAS 05003-6712, the B2 emission-line star Al 50 (Andrews $\&$ Lindsay 1964) happened to fall on the slit of the spectrograph too. Its spectrum is characterised by bright emission lines from the hydrogen Balmer series and helium, superimposed on a hot continuum (Fig. 7).

\subsection{Modelling the spectral energy distributions}

The spectral energy distribution (SED) of each object was reproduced with the dust radiative transfer model DUSTY (Ivezić et al. 1999), which was made to calculate the radial density distribution consistent with radiation-driven wind theory (Ivezic $\&$ Elitzur 1995). This takes into account the dependence of the wind speed on the stellar parameters luminosity and metallicity (via the dust-to-gas mass ratio), and the drift speed between the dust and the gas. This formalism is confirmed to hold for $\mathrm{OH} / \mathrm{IR}$ stars in the LMC for which the wind speeds have been measured from the $\mathrm{OH}$ maser emission profiles (Marshall et al. 2004), a few of which are in common with the sample we discuss here. Some of the model parameters that we will analyse in detail are summarised in Table 4. We discuss some of the input parameters and the reliability of the model output, before analysing the results.

\subsubsection{Stellar effective temperatures}

The stellar effective temperatures corresponding to the M-type spectral subclasses are taken from Fluks et al. (1994). These are strictly speaking valid for luminosity class III giants of solar abundances, and differences may be expected for metal-poor stars and for luminous supergiants. The stratification of the extended molecular atmosphere together with variations over the pulsation cycle limit the accuracy with which these cool giants may be assigned a value for the effective temperature. Where more determinations of the spectral type were available and/or classifications to half a subclass were given, the spectral subclasses were averaged and the stellar effective temperature was obtained by linear interpolation of the given values. The thus adopted values are probably only accurate to $\sim 300 \mathrm{~K}$ (cf. Houdashelt et al. 2000). We used the synthetic spectra from Fluks et al. closest to the actual object's spectral type as models for the external radiation field when running the DUSTY code.

Despite the prominent and unique molecular absorption features characterising carbon star spectra, it is remarkably difficult to classify these spectra and even more difficult to assign stellar effective temperatures. We constructed a linear grid of stellar effective temperature values between $2200 \mathrm{~K}$ for spectral type $\mathrm{C} 10$ and $4000 \mathrm{~K}$ for $\mathrm{C} 0$. We stress that this is a purely ad hoc temperature scale, and we will limit the quantitative interpretation of the carbon stars in our sample, but it nevertheless allows to rank the carbon stars according to their temperatures and study the qualitative effect that differences in the surface temperature may have on the circumstellar envelope and massloss rate. Bergeat et al. (2001) construct a photometric temperature classification scheme, and it would be useful to tally their findings with a spectral classification scheme. In running DUSTY we assumed a blackbody of the effective temperature as the model for the external radiation field. 


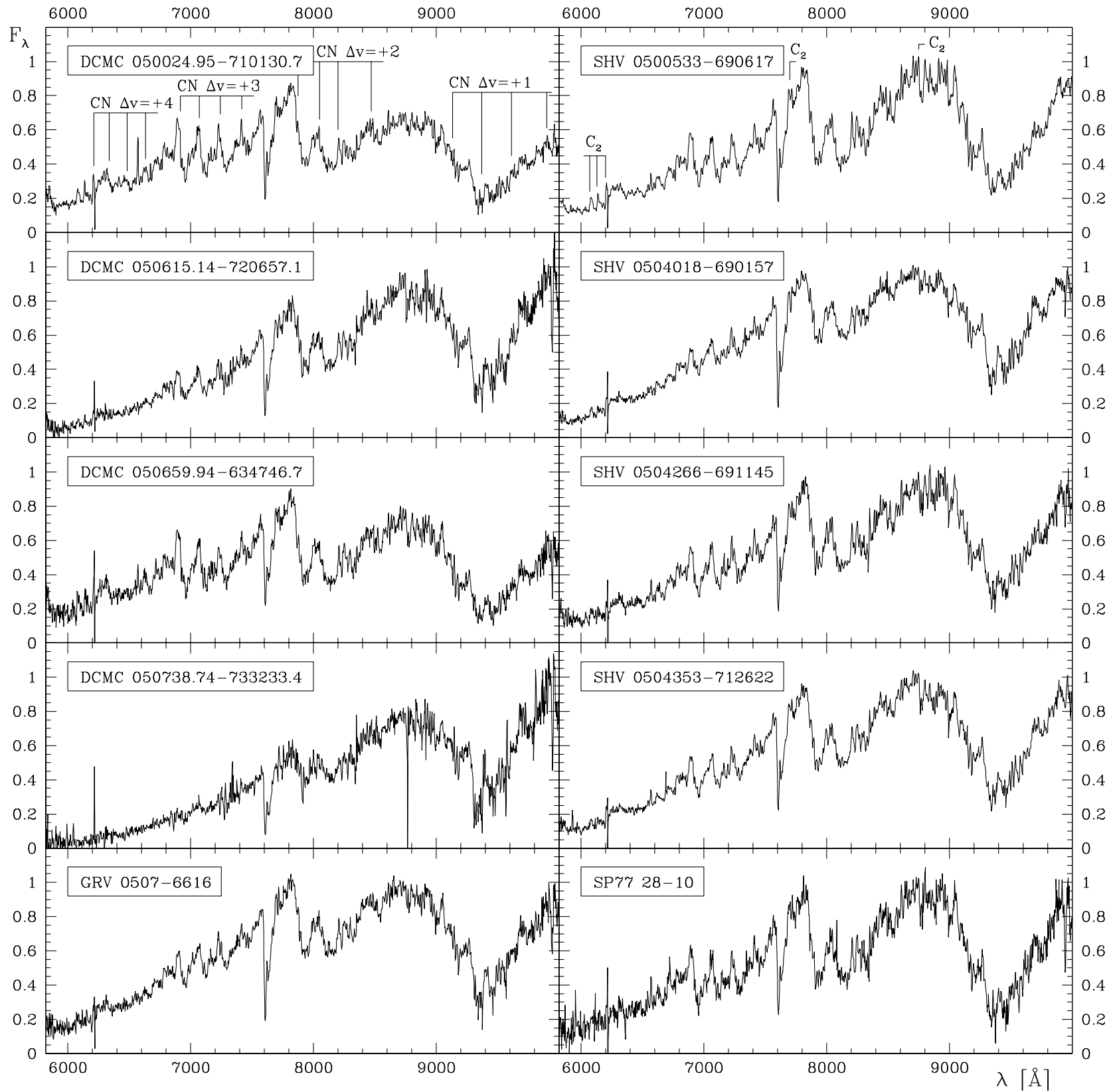

Fig. 4. EMMI spectra of carbon stars.

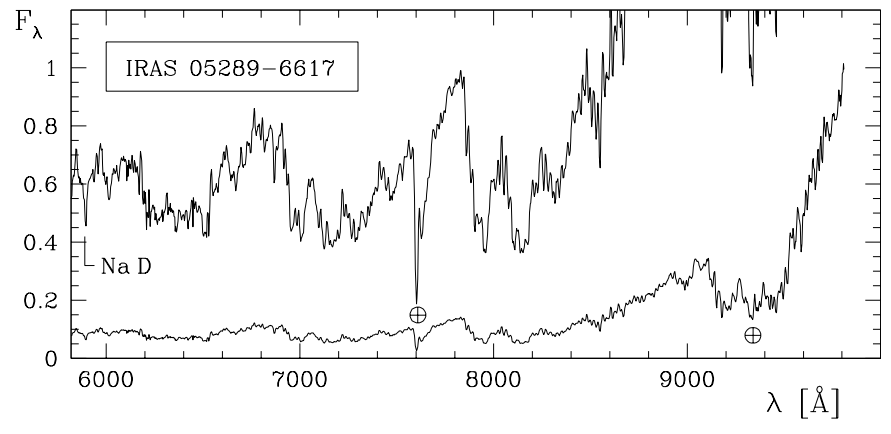

Fig. 5. EMMI spectrum of the carbon star IRAS 05289-6617.

For MS and S-type stars things become even more uncertain. We assign stellar effective temperatures in between the values we would have given were they of either M-type or C-type. For the purpose of running DUSTY, we assumed that the spectral energy distribution of the external radiation field were that of an equivalent M-type synthetic spectrum from Fluks et al. (1994).

\subsubsection{Grain properties}

Because of the limitations of the data to constrain the dust grain properties (size, shape, composition...), we have restricted ourselves in the choice of dust type, and assumed a grain size of $0.1 \mu \mathrm{m}$ unless the fit of the DUSTY model to the observed SED improved notably by making a different choice. In order to derive the total mass budget one also has to adopt a mass density for the grains. Although this varies with the type of 


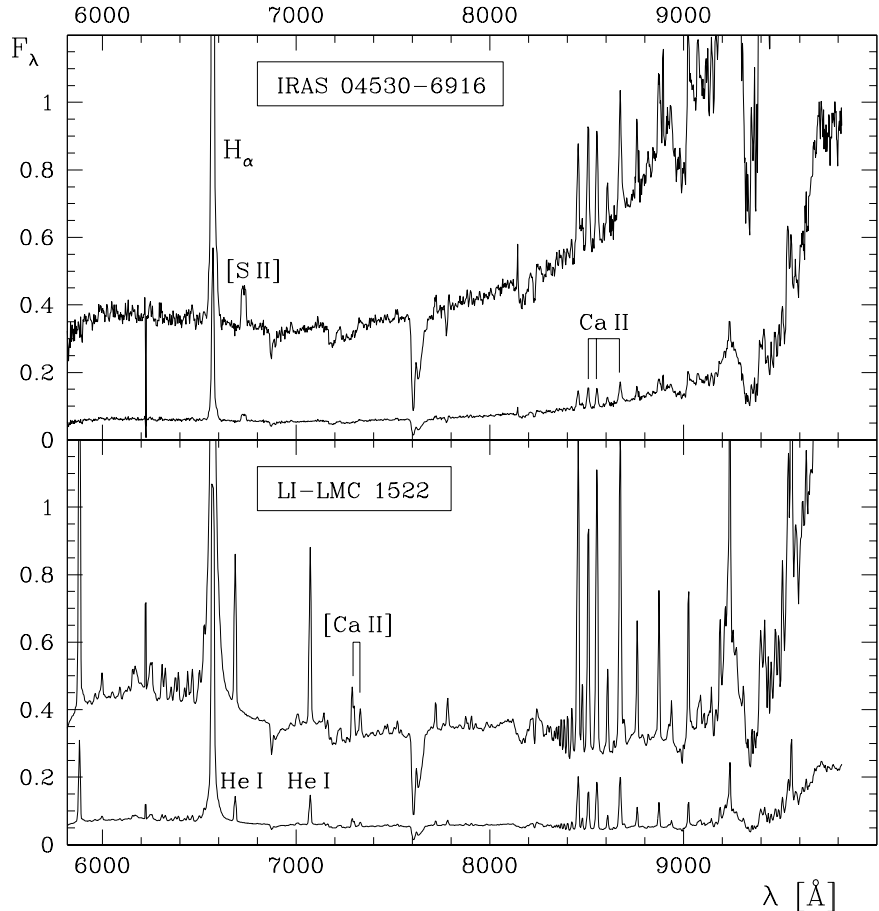

Fig. 6. EMMI spectra of two dusty emission-line objects.

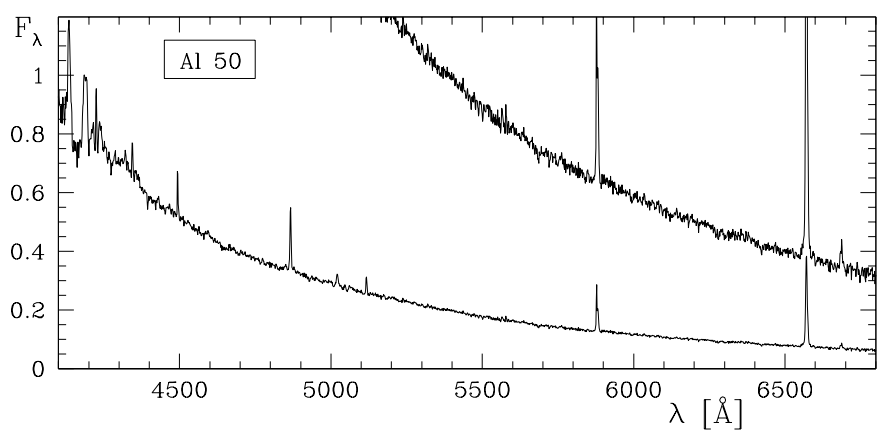

Fig. 7. DFOSC spectrum of the emission-line object Al50.

material the grains are composed of, we adopt a default value of $\rho_{\text {grain }}=3 \mathrm{~g} \mathrm{~cm}^{-3}$.

For oxygen-rich M-type stars and MS and S-type stars we adopted the astronomical silicate from Draine \& Lee (1984), but for the carbon stars we took different mixtures of graphite (Draine \& Lee 1984), amorphous carbon (Henning \& Mutschke 1997) and silicon carbide ( $\mathrm{SiC}$, from Pégourié 1988). By default we used amorphous carbon only, but DCMC J050738.74-733233.4, SHV 0500533-690617 and SHV 0504353-712622 required the inclusion of 10 per cent of $\mathrm{SiC}$, and the ISO spectrum of IRAS 05289-6617 suggests the need of 20 percent of SiC (van Loon et al. 1999b). The SEDs of DCMC J050024.95-710130.7 and DCMC J050615.14-720657.1 are better reproduced by taking only 50 per cent of amorphous carbon, with the remainder made up with graphite or with 40 per cent graphite and 10 per cent $\mathrm{SiC}$, respectively.

The near-IR extinction and mid-IR emission of several amongst the most dust-enshrouded M-type stars of our sample could be better brought in line with each other by reducing the grain size to $0.06 \mu \mathrm{m}$ (IRAS 5128-6455) or $0.05 \mu \mathrm{m}$ (IRAS 04407-7000, IRAS 04498-6842 and WOH G64), or by taking a standard MRN size distribution (a power-law with exponent -3.5; Mathis et al. 1977) between a minimum grain size of $0.01 \mu \mathrm{m}$ and a maximum grain size of $0.1 \mu \mathrm{m}$ (IRAS 05003-6712). Amongst the carbon stars we deviated from the $0.1 \mu \mathrm{m}$ grain size only in the case of SHV 0504353-712622 $(0.01 \mu \mathrm{m})$, and IRAS 5289-6617 for which we adopted an MRN distribution of larger grains between 0.1 and $1 \mu \mathrm{m}$. The latter object has both a cool photosphere and abnormally cool dust, and with the high fraction of $\mathrm{SiC}$ (see above) it is also mineralogically peculiar.

Models were first computed for a dust temperature at the inner radius of the circumstellar envelope of $T_{\text {dust }}=1000$ to $1200 \mathrm{~K}$, but in many cases an acceptable fit to the observed SED could only be achieved by lowering this temperature significantly - although altering the grain properties may also have the desired effect.

\subsubsection{Luminosities}

To derive the bolometric luminosities the DUSTY output SED needs to be scaled to fit the observed SED. Adopting a distance to the LMC of $50 \mathrm{kpc}$, this scaling factor then yields the bolometric luminosity under the assumption that interstellar extinction is negligible over most of the infrared SED.

All objects are long-period variables. Although the amplitude of variability drastically diminishes at IR wavelengths, it can still reach a magnitude in bolometric luminosity for AGB stars (for supergiants the amplitude is usually smaller in units of magnitude even if it corresponds to a larger change in energy output). This variability is reflected in differences between the DENIS and 2MASS near-IR magnitudes, and between the MSX, ISO and IRAS mid-IR flux densities. We attempt to fit the DUSTY model to a roughly "mean" SED. Apart from the uncertainty in the distance to the LMC of around 10 per cent, the values for the luminosities of individual stars are believed to be accurate to within $\sim 20$ per cent.

\subsubsection{Mass-loss rates}

In order to obtain total (gas+dust) mass-loss rates one has to know the gas-to-dust mass ratio in the outflow. In the absence of knowledge thereof we assume a value of $\psi=\rho_{\text {gas }} / \rho_{\text {dust }}=$ 500 , which we justify by scaling the typical value of $\psi=200$ for giants with solar abundances to the lower metallicity of the LMC (cf. van Loon et al. 1999b). For most of the dustenshrouded objects it would seem plausible that the dust condensation process reaches its maximum efficiency, supporting these low gas-to-dust ratios, but for less evolved and/or hotter objects this may no longer be the case, possibly leading to underestimated values for their mass-loss rates.

Many of the objects in our sample are detected in the IRAS data at $60 \mu \mathrm{m}$, at a level much higher than can be reconciled with a constant mass-loss rate model. Although these detections need to be confirmed at a higher sensitivity and angular resolution, possible explanations could include the 
heating of interstellar dust by the supergiants and/or ancient episodes of enhanced mass loss. The latter is expected especially for AGB stars descending from low-mass (1-3 $\left.M_{\odot}\right)$ Main-Sequence progenitors, as the effects of thermal pulses on the upper AGB on the surface temperature and luminosity are much more dramatic than for their more massive siblings (cf. Schröder et al. 1999).

There is ample opportunity for making different choices of grain properties, dust temperature, optical depth etcetera without affecting the fit of the DUSTY model to the SED. This is especially true for objects with optically thin dust shells. However, fortunately the resulting mass-loss rate is surprisingly little influenced by this degeneracy, thanks to the scaling inter-relationships between the quantities that govern the radiative transfer (Elitzur \& Ivezić 2001; cf. van Loon et al. 1997). Apart from largely systematic uncertainties such as the gas-todust ratio, the values for the mass-loss rates of individual objects are reliable to within a factor of $\sim$ two. This is confirmed by the observed spread in the $L, T_{\text {eff }}$ dependence of the massloss rate (see below).

\section{Mass-loss rate as a function of bolometric luminosity and stellar effective temperature}

\subsection{Mass-loss rates from oxygen-rich dust-enshrouded red giants in the Large Magellanic Cloud}

The mass-loss rates reached by more luminous AGB stars are higher than those reached by less luminous AGB stars, but there is a lot of intrinsic scatter in the mass-loss rate versus luminosity relation due to evolutionary effects (Fig. 8). Stars that have only just reached the thermal pulsing stage of AGB evolution do not experience as high a mass-loss rate as when they reach the AGB tip and develop a superwind. Likewise, although red supergiants feature mass-loss rates that are comparable to those reached by luminous tip-AGB stars, they do not seem to continue the trend of the mass-loss rate increasing with luminosity because their true superwind stage lasts very briefly and thus this evolutionary phase is largely missed. The one exception within our sample is WOH G64, the most luminous red supergiant in the Magellanic Clouds with the largest mass-loss rate.

Our sample is obviously biased against the most severely dust-enshrouded stars for which no spectrum of the stellar photosphere can be taken at optical wavelengths. This is especially true for the carbon stars, of which the circumstellar dust envelopes become optically thick relatively easily as a result of the higher opacity of carbon-rich dust and the more compact geometry of the envelope (cf. Fig. 14). Hence our sample seems to have fewer examples of the extremely high mass-loss rates as compared with the sample of obscured objects in van Loon et al. (1999b) and their relationships for the classical mass-loss rate limit and mass-loss rate limit in the presence of multiple scattering of photons off dust grains (the long and short-dashed lines in Fig. 8, respectively). Nonetheless, our sample does include several $\mathrm{OH} / \mathrm{IR}$ stars which are in the superwind phase, and our optical spectroscopy of these heavily dust-enshrouded objects makes a significant contribution to probing the

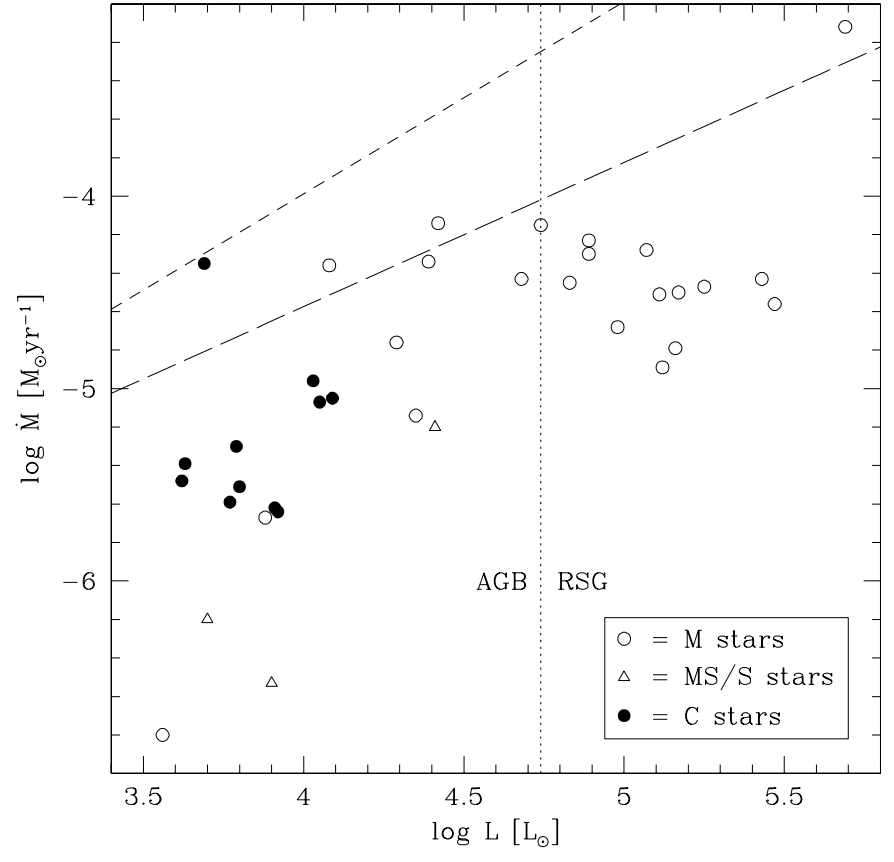

Fig. 8. Mass-loss rate as a function of bolometric luminosity, for M-type stars (circles), MS or S-type stars (triangles), and carbon stars (solid dots). The classical AGB luminosity limit is marked by a vertical dotted line. The long and short-dashed slanted lines mark the classical and multiple-scattering limits to the mass-loss rate (van Loon et al. 1999b).

stellar parameters well into this literally obscure phase of stellar evolution.

At least part of the evolutionary effect in the dispersion of mass-loss rates can be related to the stellar photospheric temperature. On the AGB, stars evolve not only to become brighter but also to become cooler, whilst red supergiants first cool rapidly but are then expected (on the basis of models) to increase in luminosity somewhat at an essentially constant temperature. It is not clear at what precise evolutionary stage we capture the red supergiants in our sample.

When the mass-loss rate is held against the stellar effective temperature, however, no clear trend is seen (Fig. 9). The M-type stars with the lowest mass-loss rates are fairly warm and the coolest M-type stars have relatively high mass-loss rates, but the red supergiants trouble this picture as they have high mass-loss rates not because they are cool (they are, in fact, amongst the warmer stars in our sample) but simply because they are luminous. This might give the impression that the luminosity dependence of the mass-loss rate dominates over the temperature dependence. However, it should be realised that the range in luminosities is vastly larger (two orders of magnitude) than the range in stellar effective temperature (less than a factor two) within our sample.

A surface can be fit to the $\dot{M}\left(L, T_{\text {eff }}\right)$ plane for the M-type stars, with useful constraints on the fit parameters. In practice, we fit a flat surface in logarithmic space using a non-linear least-squares Marquardt-Levenberg algorithm. We thus derive:

$\log \dot{M}=\alpha+\beta \log \left(\frac{L}{10000 L_{\odot}}\right)+\gamma \log \left(\frac{T_{\mathrm{eff}}}{3500 \mathrm{~K}}\right)$, 


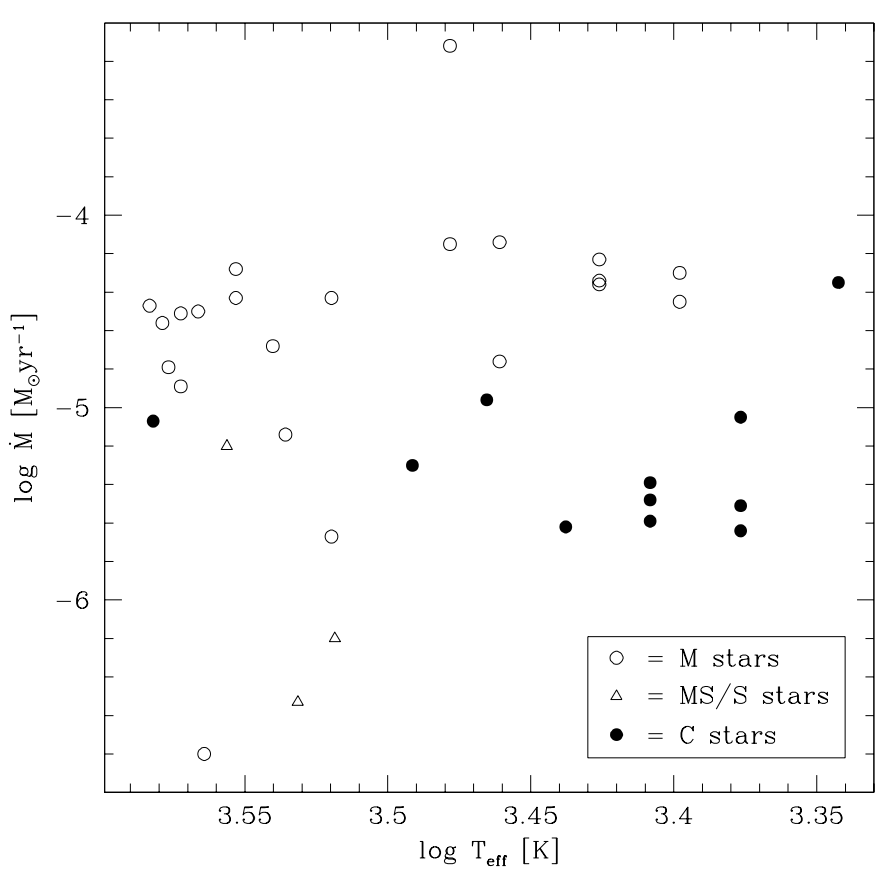

Fig. 9. Mass-loss rate as a function of stellar effective temperature. Symbols are the same as in Fig. 8.

with for M-type stars: $\alpha=-5.65 \pm 0.15, \beta=1.05 \pm 0.14$ and $\gamma=-6.3 \pm 1.2$.

This solution for the mass-loss rate recipe features an approximately linear increase of the mass-loss rate with an increase in luminosity, which is consistent with the radiationdriven dust wind theory including multiple-scattering (cf. van Loon et al. 1999b). This is also reproduced for a set of synthetic stars by Schröder et al. (2003), who find a luminosity dependence with an exponent of 1.16.

Besides the luminosity dependence, the mass-loss rate is also seen to depend sensitively on the stellar effective temperature, approximately inversely as the sixth power. A strong temperature dependence was initially suggested by Arndt et al. (1997) on the basis of models for carbon stars. Although they found an even stronger dependence of the mass-loss rate on the stellar effective temperature, with an exponent of -8.3 , their updated model calculations reduce the temperature exponent to -6.8 (Wachter et al. 2002); this is fully consistent with our observed value of $-6.3 \pm 1.2$.

These authors also found a luminosity dependence of the mass-loss rate, with an exponent of 1.5 (Arndt et al. 1997) and 2.5 (Wachter et al. 2002), respectively. Although these values are significantly larger than our observed value for the luminosity exponent of $1.05 \pm 0.14$ they also include a dependence on the stellar mass, with a decreasing mass-loss rate for an increase in stellar mass. This effect counteracts upon the luminosity dependence and, if the mass dependence were somehow incorporated within the luminosity dependence, it would support a lower observed value for the luminosity exponent in the absence of an explicit parameterisation in terms of stellar mass. Our formula may thus be consistent with the models of Arndt and Wachter, but this must be tested with objects for

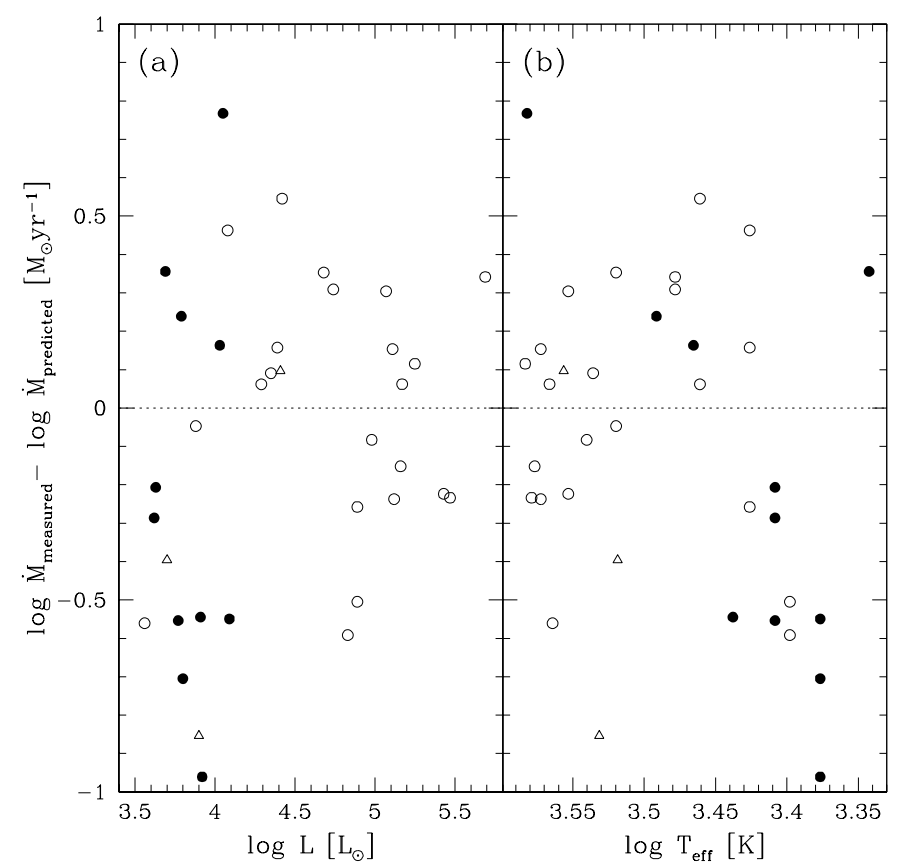

Fig. 10. Discrepancies between measured mass-loss rates from modelling of the SED, and the mass-loss rates as predicted from the $L$, $T_{\text {eff }}$ dependence of the mass-loss rate for M-type stars, plotted versus bolometric luminosity a) and stellar effective temperature b). Symbols are the same as in Fig. 8.

which the current masses are determined from accurate modelling of their pulsation characteristics.

The residuals from the $\dot{M}\left(L, T_{\text {eff }}\right)$ fit (Fig. 10) show a dispersion of only $0.3 \mathrm{dex}$ in $\log \dot{M}$ (a factor two in $\dot{M}$ ), which is comparable to the accuracy of an individual estimate of the mass-loss rate. Other stellar parameters may contribute to the spread, but it is clear that the luminosity and temperature dominate the mass-loss rate recipe for these stars.

We do not account for departures from spherical symmetry of the mass loss. Asymmetric flows are often seen in Planetary Nebulae and post-RSG objects, and thought to have their origin during the previous superwind phase. Much amplified in post-AGB and post-RSG objects due to the interaction of a fast wind with the slower superwind, it is not yet clear how prominent or common these asymmetries are in the winds of AGB stars and red supergiants. There exists no evidence for the global mass-loss rate to be affected by asymmetry in the outflow kinematics, or by the presence of a binary companion. Our sample might already include binaries or objects with asymmetric outflows as we have not wittingly excluded them - in which case the mass-loss rates for such objects cannot be different by more than a factor two.

We now divide the sample of M-type stars into red supergiants, with $\log L>4.9$, and AGB stars, with $\log L<4.9$ (see next section), and fit a similar $\dot{M}\left(L, T_{\text {eff }}\right)$ formula to each of these subsamples. Despite the smaller number of stars to constrain the fit (10 red supergiants and 12 AGB stars), the results are in excellent agreement with each other. In particular, the luminosity exponent $\beta=0.82 \pm 0.38$ for the red supergiants and $\beta=1.10 \pm 0.40$ for the AGB stars are indistinguishable. Despite the much larger uncertainty on the temperature 
exponent, where we note that the distribution of red supergiants is heavily skewed towards early types, with $\gamma=-10.8 \pm 2.7$ for the red supergiants and $\gamma=-5.2 \pm 3.0$ for the AGB stars these are also fully consistent with each other. The fits lead to an estimate for the reference mass-loss rate of $\alpha=-5.3 \pm 0.5$ and $\alpha=-5.6 \pm 0.2$ for the red supergiants and AGB stars, respectively, again suggesting that the mass-loss rates from both red supergiants and oxygen-rich AGB stars are governed by the same mechanism and in the same manner.

If the mass-loss rate recipe for M-type stars is applied to the (M)S and C-type stars, their dispersion is reduced as well, suggesting that the mass-loss rate of these types of chemically distinct objects may depend on the luminosity and stellar effective temperature in a similar fashion. Attempts to fit an $\dot{M}\left(L, T_{\text {eff }}\right)$ relation to these classes of stars separately lead to qualitative similar exponents (positive for the luminosity dependence and negative for the temperature dependence) but in view of the smaller number of stars and the uncertainty with regard to the temperature scale for these objects we prefer not to publish the results of these fits to avoid abuse.

\subsection{Comparison with galactic red giants}

We now investigate to what extent the mass-loss rate recipe is valid for other samples, by applying it to galactic AGB stars and red supergiants. Jura \& Kleinmann published studies of the mass loss from red giants in the solar neighbourhood, which provides us with three samples: (1) AGB stars pulsating with an intermediate-period (Jura \& Kleinmann 1992), (2) dustenshrouded AGB stars with a large $60 \mu \mathrm{m}$ excess (Jura \& Kleinmann 1989), and (3) red supergiants (Jura \& Kleinmann 1990). From their spatial distributions Jura \& Kleinmann estimate progenitor masses of respectively $1-1.2 M_{\odot}, 1.5 M_{\odot}$ and $>20 M_{\odot}$ for these three samples. We selected all M-type stars for which spectral sub-classification is available to allow a determination of $T_{\text {eff }}$, and only those stars for which Jura \& Kleinmann estimated a mass-loss rate: 5 intermediateperiod AGB stars (with pulsation periods of 310-372d), 26 dust-enshrouded AGB stars and 19 red supergiants. Their properties are listed in Table 5. Jura \& Kleinmann (1990) list spectral types for the supergiants, but spectral types were obtained from Simbad for the other two samples and for the red supergiant NMLCyg. The values for $T_{\text {eff }}$ are assigned according to the same calibration scheme as for our sample of Magellanic objects.

Jura \& Kleinmann (1992) estimated distances for the intermediate-period AGB stars using the $K$-band periodluminosity relation for Mira variables. For a few nearby examples these are consistent with determinations from Hipparcos data (Knapp et al. 2003). Nevertheless, Jura \& Kleinmann assumed the luminosities to be $5000 L_{\odot}$. They estimated distances for the dust-enshrouded AGB stars from the bolometric fluxes, where they assumed that they all have a luminosity of $10^{4} L_{\odot}$ (Jura \& Kleinmann 1989). For the supergiants they estimated distances in most part from cluster membership (Jura \& Kleinmann 1990). The distance ranges for the samples are $80-300$ pc, $270-1100$ pc and 200-2500 pc, respectively.
Table 5. Galactic samples of red giants (Jura \& Kleinmann 1989, 1990, 1992), with the spectral type, the stellar effective temperature, $T_{\text {eff }}$, the bolometric luminosity, $L$, and the mass-loss rates as determined by Jura \& Kleinmann, $\dot{M}_{1}$, and as predicted by our recipe, $\dot{M}_{2}$. We omitted the IRAS prefix to some of the dust-enshrouded AGB stars.

\begin{tabular}{|c|c|c|c|c|c|}
\hline 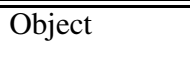 & $\begin{array}{l}\text { Spectral } \\
\text { type }\end{array}$ & $\begin{array}{c}T_{\text {eff }} \\
\mathrm{K}\end{array}$ & $\begin{array}{c}\log L \\
L_{\odot}\end{array}$ & $\begin{array}{l}\log \dot{M}_{1} \\
M_{\odot} / \mathrm{yr}\end{array}$ & $\begin{array}{l}\log \dot{M}_{2} \\
M_{\odot} / \mathrm{yr}\end{array}$ \\
\hline \multicolumn{6}{|c|}{ Intermediate-period AGB stars (Jura $\mathcal{E}$ Kleinmann 1992) } \\
\hline $\mathrm{S} \mathrm{CrB}$ & M7 & 3126 & 3.70 & -6.96 & -5.66 \\
\hline RLeo & M 8 & 2890 & 3.70 & -7.07 & -5.44 \\
\hline R LMi & M7 & 3126 & 3.70 & -6.82 & -5.66 \\
\hline RX Boo & M7.5 & 3008 & 3.70 & -6.80 & -5.55 \\
\hline W Hya & M7 & 3126 & 3.70 & -7.00 & -5.66 \\
\hline \multicolumn{6}{|c|}{ Dust-enshrouded AGB stars (Jura E Kleinmann 1989) } \\
\hline KU And & M9 & 2667 & 4.00 & -4.72 & -4.91 \\
\hline WX Psc & M9 & 2667 & 4.00 & -4.64 & -4.91 \\
\hline $02316+6455$ & M9 & 2667 & 4.00 & -5.07 & -4.91 \\
\hline $02351-2711$ & M9 & 2667 & 4.00 & -5.44 & -4.91 \\
\hline NML Tau & M6 & 3309 & 4.00 & -5.22 & -5.50 \\
\hline $04307+6210$ & M6 & 3309 & 4.00 & -5.44 & -5.50 \\
\hline TX Cam & M 8.5 & 2778 & 4.00 & -5.60 & -5.02 \\
\hline $05411+6957$ & M9 & 2667 & 4.00 & -5.23 & -4.91 \\
\hline V Cam & M7 & 3126 & 4.00 & -5.66 & -5.34 \\
\hline $06300+6058$ & M9 & 2667 & 4.00 & -5.28 & -4.91 \\
\hline GX Mon & M9 & 2667 & 4.00 & -5.03 & -4.91 \\
\hline IW Hya & M9 & 2667 & 4.00 & -5.02 & -4.91 \\
\hline V2108 Oph & M9 & 2667 & 4.00 & -5.42 & -4.91 \\
\hline V774 Sgr & M5 & 3434 & 4.00 & -5.55 & -5.60 \\
\hline 18009-2019 & M 8 & 2890 & 4.00 & -5.37 & -5.12 \\
\hline $18135-1641$ & M5 & 3434 & 4.00 & -5.30 & -5.60 \\
\hline $18204-1344$ & M 8 & 2890 & 4.00 & -5.38 & -5.12 \\
\hline V1111 Oph & M9 & 2667 & 4.00 & -5.28 & -4.91 \\
\hline $18413+1354$ & M7 & 3126 & 4.00 & -5.26 & -5.34 \\
\hline V3953 Sgr & M9 & 2667 & 4.00 & -5.17 & -4.91 \\
\hline V3880 Sgr & M 8 & 2890 & 4.00 & -5.00 & -5.12 \\
\hline V342 Sgr & M9 & 2667 & 4.00 & -5.36 & -4.91 \\
\hline $20440-0105$ & M9 & 2667 & 4.00 & -5.57 & -4.91 \\
\hline UUPeg & M7 & 3126 & 4.00 & -5.80 & -5.34 \\
\hline RT Cep & M 6 & 3309 & 4.00 & -5.60 & -5.50 \\
\hline $23496+6131$ & M9 & 2667 & 4.00 & -5.27 & -4.91 \\
\hline \multicolumn{6}{|c|}{ Red supergiants (Jura E Kleinmann 1990) } \\
\hline KW Sgr & M OIa & 3895 & 5.00 & -5.40 & -4.89 \\
\hline VXSgr & M 4Ia & 3574 & 5.18 & -4.40 & -4.47 \\
\hline UY Sct & M 4Iab & 3574 & 5.00 & -5.15 & -4.66 \\
\hline $\mathrm{BD}+24^{\circ} 3902$ & M 1Ia & 3810 & 5.00 & -6.00 & -4.83 \\
\hline BI Cyg & M 4Ia & 3574 & 5.30 & -5.22 & -4.34 \\
\hline KY Cyg & M3.5Ia & 3620 & 5.60 & -5.40 & -4.06 \\
\hline NML Cyg & M 6III & 3309 & 5.70 & -4.00 & -3.71 \\
\hline$\mu \mathrm{Cep}$ & M 2Ia & 3736 & 5.60 & -6.05 & -4.15 \\
\hline PZCas & M 2Ia & 3736 & 5.30 & -5.00 & -4.46 \\
\hline TZCas & M 2Iab & 3736 & 5.00 & -5.52 & -4.78 \\
\hline S Per & M 3Ia & 3666 & 5.30 & -5.15 & -4.41 \\
\hline SUPer & M3Iab & 3666 & 5.00 & -5.22 & -4.73 \\
\hline$\alpha$ Ori & M 1 & 3810 & 5.00 & -6.70 & -4.83 \\
\hline VY CMa & M5I & 3434 & 5.60 & -4.00 & -3.92 \\
\hline EV Car & M 4Ia & 3574 & 5.30 & -5.22 & -4.34 \\
\hline CK Car & M3.5Iab & 3620 & 5.00 & -5.70 & -4.69 \\
\hline IX Car & M 2Iab & 3736 & 5.00 & -5.05 & -4.78 \\
\hline V396 Cen & M 4Ia & 3574 & 5.30 & -6.00 & -4.34 \\
\hline AH Sco & M4 & 3574 & 5.48 & -5.10 & -4.16 \\
\hline
\end{tabular}




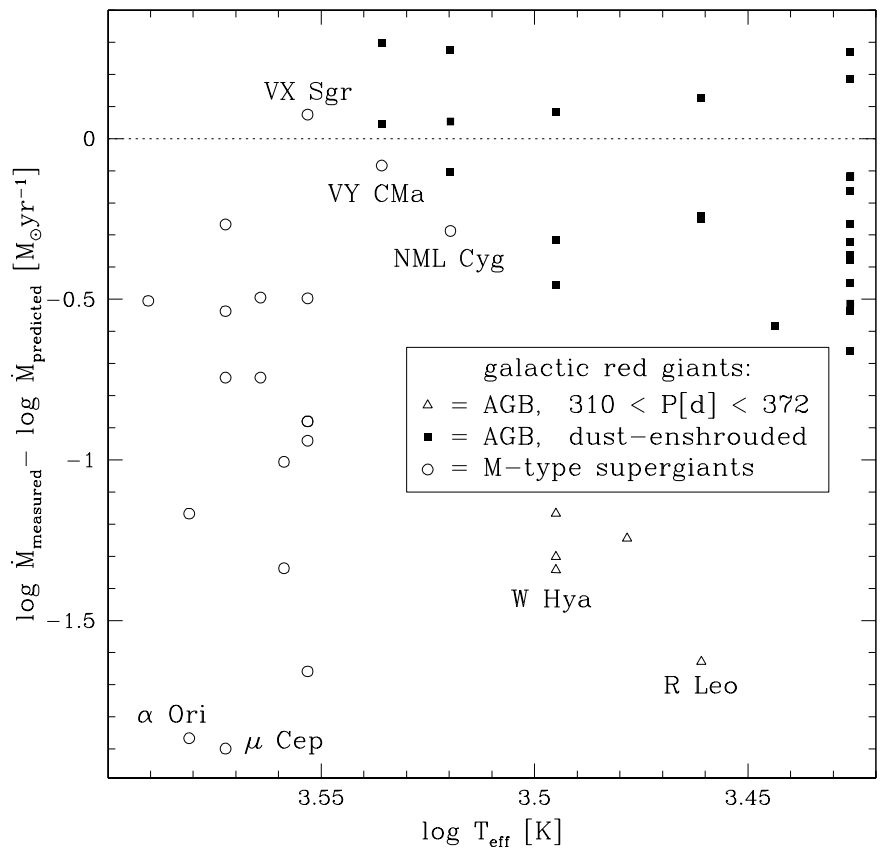

Fig. 11. Discrepancies between the mass-loss rates derived from $60 \mu \mathrm{m}$ flux densities, and the mass-loss rates as predicted from the $\dot{M}\left(L, T_{\text {eff }}\right)$ formula for dust-enshrouded stars in the LMC, plotted versus stellar effective temperature, for stars in the solar neighbourhood (Jura \& Kleinmann 1989, 1990, 1992).

The method of Jura \& Kleinmann to derive mass-loss rates, which they applied to all three samples in the same manner, is based on a scaling of the measured $60 \mu \mathrm{m}$ flux density (Jura $1986,1987)$. The result depends on distance, $d$, luminosity, $L$, and wind speed, $v$, as $\dot{M} \propto v d^{2} / \sqrt{L}$. The wind speed was measured for almost every intermediate-period and dustenshrouded AGB star that we selected, but for only 7 out of the 19 supergiants. For the stars without a direct measurement of the wind speed they assumed $v=15$ and $30 \mathrm{~km} \mathrm{~s}^{-1}$ for dustenshrouded AGB stars and supergiants, respectively. The selected intermediate-period AGB stars have a (measured) wind speed of only $v \simeq 6 \mathrm{~km} \mathrm{~s}^{-1}$. Some supergiants also have a slow wind, notably $\alpha$ Ori and $\mu$ Cep $\left(v=10 \mathrm{~km} \mathrm{~s}^{-1}\right)$ and Jura \& Kleinmann (1990) suggest that this may be due to a much reduced dust-to-gas ratio $(\psi \gg 200)$. If that is the case then their values for the mass-loss rates of these stars will have been underestimated.

There is excellent agreement between the mass-loss rates as predicted using our recipe and the measured Jura \& Kleinmann mass-loss rates for the galactic dust-enshrouded AGB stars (Fig. 11, solid squares), where most of the scatter is due to deviations from the assumed luminosity of $10^{4} L_{\odot}$ for these stars. We note that the sample of dust-enshrouded AGB stars in Jura \& Kleinmann (1989) includes another 5 more extreme oxygenrich objects without spectral sub-classification, for which they estimate mass-loss rates between $2 \times 10^{-5}$ and $2 \times 10^{-4} M_{\odot} \mathrm{yr}^{-1}$. A similar bias is present in our Magellanic sample (see next section). The good agreement between the dust-enshrouded (oxygen-rich) AGB stars in the LMC and in the solar neighbourhood suggests that there is no evidence for the total massloss rates for these classes of stars to depend on the initial metal abundance, a conclusion which was already reached by van Loon (2000).

Our recipe also correctly predicts the mass-loss rates for the most extreme red supergiants, notably NML Cyg, VX Sgr and VY CMa that have mass-loss rates of the order $10^{-4} M_{\odot} \mathrm{yr}^{-1}$ (Fig. 11, circles). However, most of the red supergiants from the Jura \& Kleinmann (1990) sample have measured mass-loss rates that are significantly lower than the predicted values by about a factor 3-50 (although most are within a factor 10 of the predictions). This can be due to invalidity of the massloss recipe or the assumptions made in the measured values. We already mentioned that the most discrepant objects in our comparison, $\alpha$ Ori and $\mu$ Cep have anomalously slow winds and probably a low dust-to-gas ratio. Furthermore, these two stars are the nearest supergiants in the sample and they are spatially resolved at $60 \mu \mathrm{m}$, which may have led to a further underestimate of the mass-loss rate. The same may be true to a lesser extent for the other supergiants.

There is a clear discrepancy for the intermediate-period AGB stars (Fig. 11, triangles), with the predictions exceeding the measured values by a factor $10-40$. These are nearby objects and the $60 \mu \mathrm{m}$ flux may again have been underestimated. They are cool and pulsate vigorously: 4 out of 5 are Mira-type variables whilst W Hya is a semi-regular variable. But their periods are with $P<400 \mathrm{~d}$ shorter than those of the dust-enshrouded stars, supergiants and the Magellanic sample. They are also optically bright. Our recipe is not meant to be applied to these stars as it was derived for dust-enshrouded objects with $P>400 \mathrm{~d}$. The dust formation in the intermediateperiod AGB stars may not be at its maximum efficiency, resulting in lower dust-to-gas ratios (explaining their slow winds). Perhaps the different pulsation properties or the higher gravity near the photospheres of these stars causes the atmosphere to be elevated less than in more extreme objects. Indeed, circumstellar dust envelopes are much more prominent for objects with $P>400$ d than for objects with $P<400$ d (e.g., Knapp et al. 2003).

\section{The Hertzsprung-Russell diagram of dust-enshrouded AGB stars and red supergiants}

Now that we have determined the bolometric luminosities and stellar effective temperatures for the objects in our sample, we can place them in a physical Hertzsprung-Russell diagram (Fig. 12). This allows a comparison with theoretical evolutionary tracks to be made. To this purpose, the tracks from Bertelli et al. (1994) are plotted, for a typical LMC metallicity of $[\mathrm{Fe} / \mathrm{H}]=-0.4$.

Many of the stars in our sample are cooler than the endpoints on the AGB or RSG tracks. Indeed, despite the low metal abundance in the LMC, very late spectral types are encountered, with now at least two M 10 stars known on the AGB and for instance the red supergiant WOHG64 also being much cooler than the evolutionary models produce (cf. Massey \& Olsen 2003). The late-type AGB stars in our sample are not even the most extreme objects; more heavily obscured OH/IR stars such as IRAS 05298-6957 


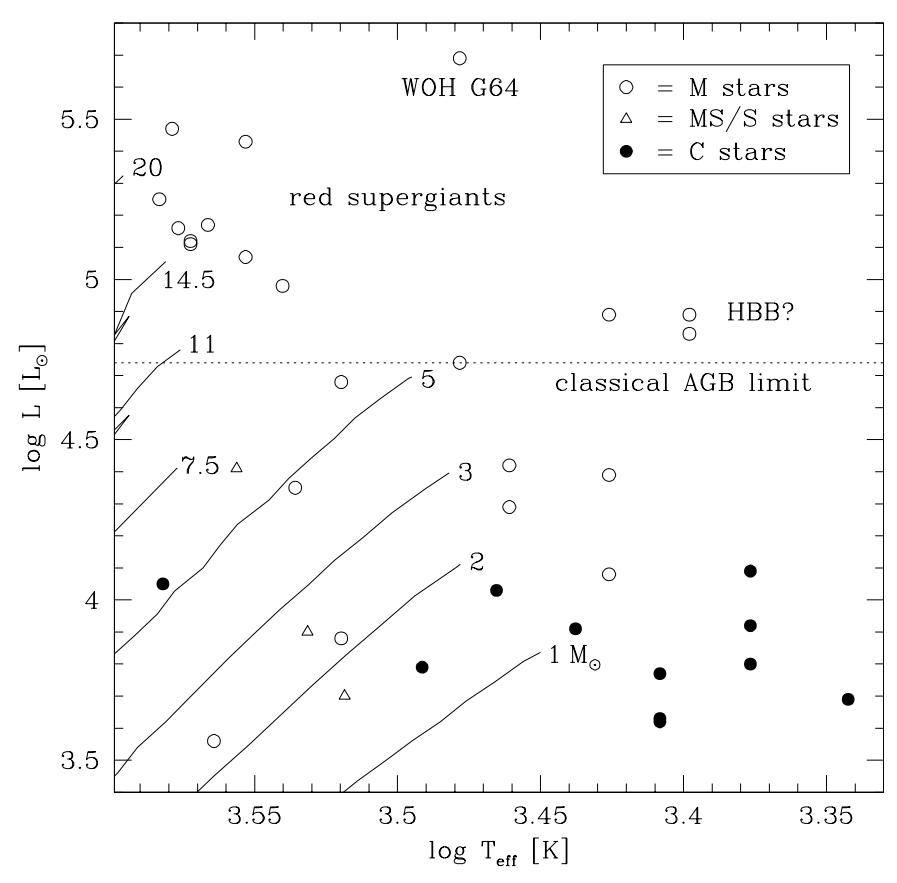

Fig. 12. The Hertzsprung-Russell diagram of bolometric luminosity versus stellar effective temperature (symbols are the same as in Fig. 8). The classical AGB luminosity limit is marked by a horizontal dotted line. Three cool stars just above this limit may be AGB stars whose luminosity is enhanced as a result of Hot Bottom Burning (HBB). Evolutionary tracks from Bertelli et al. (1994) are plotted and labelled by their Main Sequence progenitor mass.

(Wood et al. 1992; van Loon et al. 2001a) exist, for which no optical spectrum has yet been possible to take.

On the basis of the evolutionary tracks, the classical AGB luminosity limit and the distribution of the stars in the diagram, it seems a-posteriori fairly obvious which stars are intermediate-mass AGB stars and which are more massive supergiants. Rather than interpreting the three cool stars just above the classical AGB limit (Fig. 12) as much cooler than expected supergiants, it seems more logical to interpret these as slightly overluminous tip-AGB stars as there is an accepted physical mechanism for doing so: Hot Bottom Burning (HBB). The extra luminosity is generated from nuclear burning at the bottom of the deep convective zone in massive $\left(z 4 M_{\odot}\right)$ AGB stars (Boothroyd \& Sackmann 1992).

The location of the (M)S-type and carbon stars is as expected. Note that the Bertelli et al. (1994) tracks do not account for the molecular opacity efects on the atmospheres of carbon stars which are, however, incorporated in more recent models by Marigo (2002). We should also emphasize that especially the estimated temperatures of the (M)S and C-type stars are uncertain in a systematic way, and that in particular the positions of the warmest MS-type star (HV 12070) and the warmest carbon star (DCMC J050738.74-733233.4) in the diagram may need to be corrected towards lower temperatures - otherwise the implied Main-Sequence progenitor mass of 5-6 $M_{\odot}$ seems inexplicably large. These two objects have spectra that are consistent with a $\mathrm{C} / \mathrm{O}$ ratio (very) close to unity which would cause a reduced opacity in the photosphere.

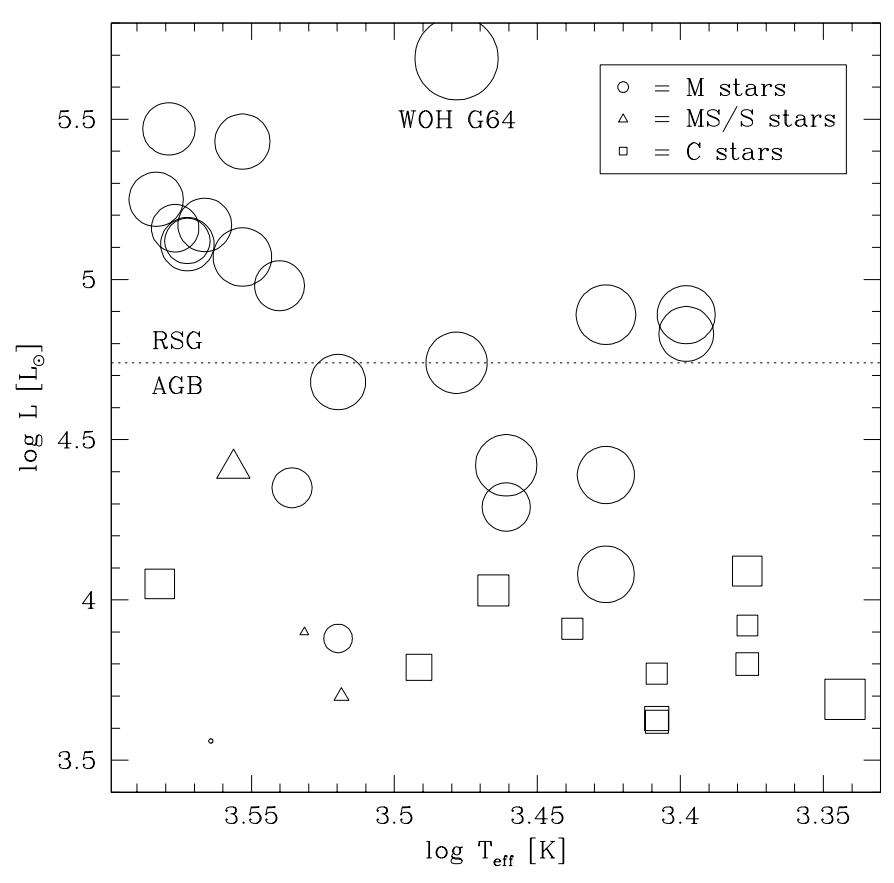

Fig. 13. The Hertzsprung-Russell diagram for the M-type stars (circles), MS or S-type stars (triangles), and carbon stars (squares), where the sizes of the symbols are logarithmically proportional to the massloss rate.

Notwithstanding this, it is clear from the diagram (Fig. 12) that the carbon stars in our sample are descended from low-mass Main Sequence stars (mostly in the range of roughly 1-2 $M_{\odot}$ ) whereas the M-type AGB stars have more massive progenitors (typically $3-5 M_{\odot}$ ). This is as expected, as HBB prevents the formation of massive carbon stars (Boothroyd \& Sackmann 1993; cf. van Loon et al. 2001a; Bergeat et al. 2002; Marigo et al. 2003). S-type stars (including stars of MS or CS-type) are thought to represent an intermediate phase in the formation of a carbon star from an oxygen-rich star earlier on the AGB (Lloyd Evans 1983). It is therefore not surprising that the (M)S-type stars in our sample are relatively warm and similarly luminous as the carbon stars (i.e. fainter than most M-type stars in our sample).

The mass-loss rate can be seen to vary across the Hertzsprung-Russell diagram due to the evolution of the stars and the dependence of the mass-loss rate on luminosity and stellar effective temperature (Fig. 13). The (M)S-type stars and the relatively warm and faint M-type AGB stars have the lowest mass-loss rates in this diagram, whilst the coolest and most luminous carbon stars and especially M-type AGB stars and red supergiants experience the most intense mass loss. This illustrates the validity of the $\dot{M}\left(L, T_{\text {eff }}\right)$ recipe we derived earlier from these data.

There is a clear tendency for the dust shells to be warmer for cooler stellar photospheres (Fig. 14). Although this may first seem odd, it can probably be understood by the dust formation region moving in closer to the star as the envelope becomes denser (or vice versa): the dust formation radius, $R_{\text {dust }}$, expressed in stellar radii, $R_{\star}$, is related to the dust temperature at the inner edge of the circumstellar dust envelope as $R_{\text {dust }} / R_{\star} \propto$ $\left(T_{\text {eff }} / T_{\text {dust }}\right)^{2}$. The objects with the relatively coldest dust and 


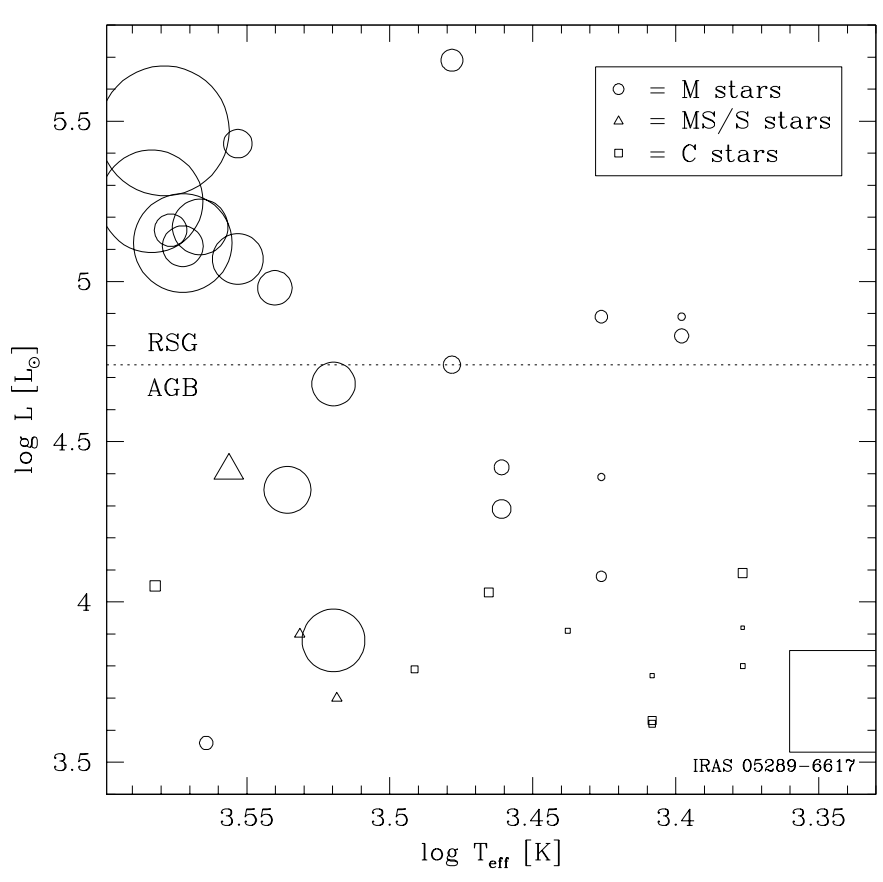

Fig. 14. Same as Fig. 13, but now the sizes of the symbols are proportional to $\left(T_{\text {eff }} / T_{\text {dust }}\right)^{2}$, which is a measure of the distance to the star of the dust formation region, $R_{\text {dust }} / R_{\star}$.

largest dust-free inner cavity are the early-M type supergiants and the coolest and most obscured carbon star in our sample, IRAS $05289-6617\left(T_{\text {eff }} \simeq 2200 \mathrm{~K}\right.$ and $\left.T_{\text {dust }} \simeq 220 \mathrm{~K}\right)$. The latter may have temporarily reduced its mass-loss rate as a result of a thermal pulse or (less likely given the short timescale) if it has just become a post-AGB object.

The fact that the warm and dense circumstellar dust envelopes surround the objects with the larger mass-loss rates and cooler stellar photospheric temperatures is not surprising, as both the mass-loss rate and the cooling of the stellar photosphere result from the elevation of the atmospheric layers of the star due to the strong radial pulsations which develop on the thermal pulsing AGB and during the red supergiant stage. This may explain the remarkable strength of the molecular bands seen in the optical spectra of dust-enshrouded M-type AGB stars as well as in the optical and near-IR spectra of dustenshrouded carbon stars in the LMC (van Loon et al. 1999a; Matsuura et al. 2002, 2005).

\section{Summary}

We presented new optical spectra for a sample of red supergiants and both oxygen-rich and carbon-rich dust-enshrouded AGB stars in the LMC. Some of these AGB stars have extremely cool stellar photospheres, with spectral types as late as M 10, despite their low metallicity. This is cooler than expected on the basis of stellar evolutionary models. The same is found for the red supergiants.

We complemented these new temperature determinations with similar LMC objects from the literature for which spectral subclassifications are available, as well as with infrared photometry to sample their spectral energy distributions that were then modelled with the radiative transfer code DUSTY to measure the mass-loss rates. The mass-loss rates were found to increase with increasing luminosity and decreasing stellar effective temperature. For the oxygen-rich, M-type AGB stars and red supergiants these dependencies were quantified by the mass-loss rate formula $\log \dot{M}=-5.65+$ $1.05 \log \left(L / 10000 L_{\odot}\right)-6.3 \log \left(T_{\text {eff }} / 3500 \mathrm{~K}\right)$, with no evidence for a difference between the red supergiants and oxygen-rich AGB stars. This formula prescribes the mass-loss rate to within a factor two for oxygen-rich stars near the tip of their AGB evolution or during the red supergiant phase when they experience strong and regular pulsation and enshroud themselves in dusty envelopes, and is valid in particular for the superwind phase of extreme mass loss. It thus complements the large body of existing formulae derived for less massive and less evolved red giants, and can be used in models for stellar and galactic evolution in stead of extra-polations of those formulae.

Application of our formula to samples of galactic oxygenrich red giants shows excellent agreement between our predictions and mass-loss rates derived from $60 \mu \mathrm{m}$ flux densities for dust-enshrouded AGB stars and the most extreme red supergiants. This suggests that the formula is valid for stars with masses from $M_{\text {ZAMS }} \sim 1.5 M_{\odot}$ to $M_{\text {ZAMS }}>20 M_{\odot}$, and that the total (gas+dust) mass-loss rate does not depend sensitively on the initial metal abundance. On the other hand, our formula predicts higher rates than the observational estimates for less extreme red giants. This failure may indicate that our formula should only be applied to dust-enshrouded objects where the dust condensation efficiency is maximal, but it may also be explained by errors in the observational estimates if the dust-togas ratio in these less extreme objects is lower than what is commonly assumed.

Carbon stars and stars of type MS or S seem to show a similar behaviour of the mass-loss rate as a function of luminosity and temperature, but a quantification of these dependencies awaits a more careful and extensive analysis of these classes of objects.

We presented Hertzsprung-Russell diagrams, which not only illustrate the trend of mass-loss rate with luminosity and temperature but also a trend for dust to form at relatively greater distances from early-M type stars than in the case of stars with cooler stellar photospheres. These diagrams also showed evidence for Hot Bottom Burning preventing carbon star formation for massive AGB stars and enhancing their luminosity at the tip of the AGB.

Acknowledgements. We are indebted to ESO for the generous allocation of Director's Discretionary Time to take the 1996 and 1998 NTT/EMMI spectra. We would like to thank the anonymous referee for helping us clarify the presentation of our manuscript and for encouraging us to test our mass-loss rate recipe on galactic stars. This research has made use of the SIMBAD database, operated at CDS, Strasbourg, France.

\section{Appendix A: Spectra of galactic M-type stars}

During the DFOSC 1995 and EMMI 2000 runs, spectra were taken of several galactic M-type stars for reference purposes. The observations were performed and reduced in exactly the same way as the LMC targets, but with exposure times ranging 
Table A.1. Spectroscopic targets in the Milky Way, listed in order of increasing Right Ascension (J2000 coordinates) and accompanied by the observing run code (either "D5" = DFOSC 1995, or "E0" = EMMI 2000). Also listed are their spectral classifications and near-IR magnitudes and mid-IR flux densities (in Jy, with the wavelengths in $\mu \mathrm{m}$ ).

\begin{tabular}{lccc|cc|rrr|rrrr}
\hline \hline \multirow{2}{*}{ Object } & \multirow{2}{*}{ RA } & \multirow{2}{*}{ Dec } & \multirow{2}{*}{ Run } & \multicolumn{2}{|c|}{ Spectral type } & \multicolumn{3}{c|}{ 2MASS } & \multicolumn{4}{c}{ IRAS } \\
& & & & Here & Previous & 1.24 & 1.66 & 2.16 & 12 & 25 & 60 & 100 \\
\hline DI Eri & 23746.8 & -453710 & D5 & M 6 & M 5/6 III & 4.41 & 3.37 & 3.12 & 3.15 & 0.85 & 0.2 & $<0.3$ \\
DG Eri & 42041.3 & -164948 & D5 & M 4 & M 3 III & 2.99 & 2.11 & 1.85 & 8.7 & 2.37 & 0.43 & $<0.4$ \\
R Dor & 43645.5 & -620438 & D5 & M 7.5 & M 8 IIIe & -2.65 & -3.73 & -4.23 & 5190.0 & 1500.0 & 255.0 & 79.0 \\
SY Men & 43916.1 & -742142 & E0 & M 10 & M & 4.89 & 3.59 & 2.81 & 51.0 & 46.0 & 7.4 & 2.1 \\
T Lep & 50450.8 & -215416 & D5 & M 6.5e & M 6e,var & 1.19 & 0.20 & -0.27 & 162.0 & 58.0 & 9.8 & 3.5 \\
RS Vel & 92345.3 & -485200 & D5 & M 10e & M 7e & 1.29 & 0.37 & -0.18 & 197.0 & 84.0 & 15.9 & $4.0 ?$ \\
\hline
\end{tabular}

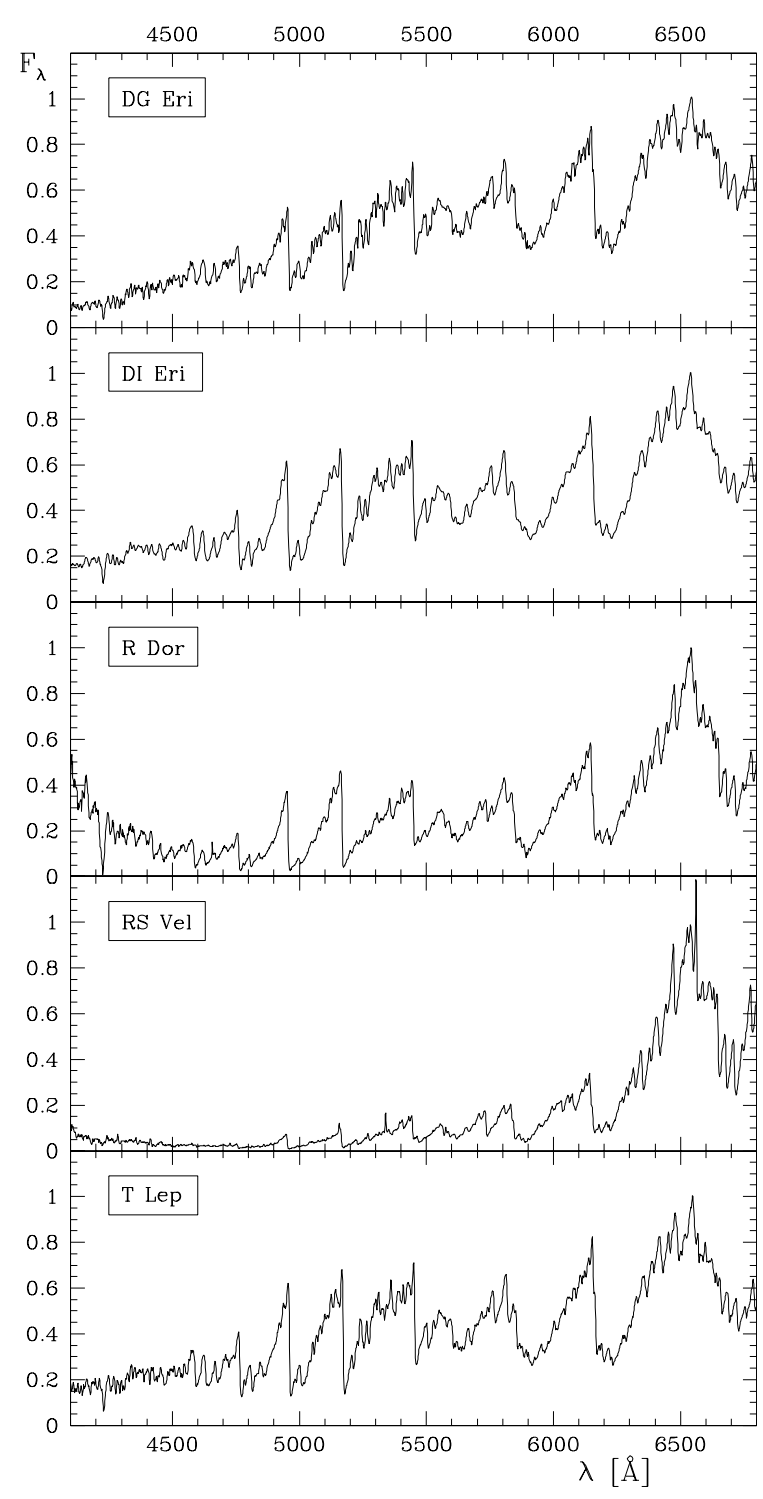

Fig. A.1. DFOSC spectra of galactic M-type stars.

between only $5 \mathrm{~s}$ and $5 \mathrm{~min}$. The spectral classification and IR photometry from 2MASS and IRAS are listed in Table A.1, and the spectra are displayed in Figs. A.1 and A.2. RS Vel was

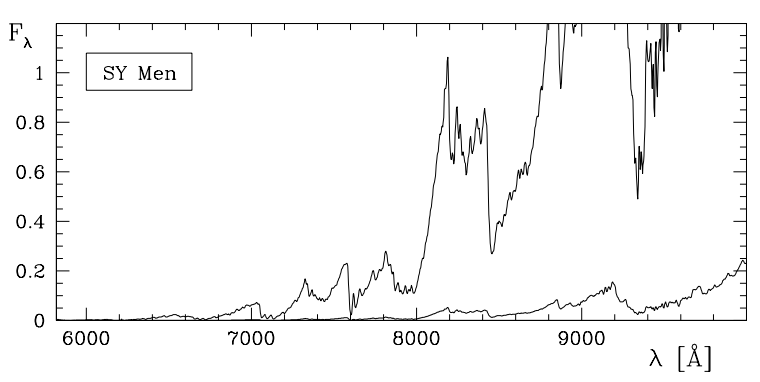

Fig. A.2. EMMI spectrum of the galactic M-type star SY Men.

also covered by MSX, yielding flux densities of $F_{8.28}=266.1$, $F_{12.13}=244.6, F_{14.65}=188.3$ and $F_{21.34}=121.5 \mathrm{Jy}$.

\section{References}

Andrews, A. D., \& Lindsay, E. M. 1964, IrAJ, 6, 241

Arndt, T. U., Fleischer, A. J., \& Sedlmayr, E. 1997, A\&A, 327, 614

Bergeat, J., Knapik, A., \& Rutily, B. 2001, A\&A, 369, 178

Bergeat, J., Knapik, A., \& Rutily, B. 2002, A\&A, 390, 967

Bertelli, G., Bressan, A., Chiosi, C., Fagotto, F., \& Nasi, E. 1994, A\&AS, 106, 275

Bessell, M. S., Wood, P. R., \& Lloyd Evans, T. 1983, MNRAS, 202, 59

Boothroyd, A. I., \& Sackmann, I.-J. 1992, ApJ, 393, L21

Boothroyd, A. I., \& Sackmann, I.-J. 1993, ApJ, 416, 762

Catelan, M. 2000, ApJ, 531, 826

Cioni, M.-R., Loup, C., Habing, H. J., et al. 2000, A\&AS, 144, 235

Cohen, M., Wheaton, Wm. A., \& Megeath, S. T. 2003, AJ, 126, 1090

Draine, B. T., \& Lee, H. M. 1984, ApJ, 285, 89

Elitzur, M., \& Ivezić, Ž. 2001, MNRAS, 327, 403

Filippenko, A. V. 1997, ARA\&A, 35, 309

Fluks, M. A., Plez, B., Thé, P. S., et al. 1994, A\&AS, 105, 311

Fouqué, P., Chevallier, L., Cohen, M., et al. 2000, A\&AS, 141, 313

Henning, Th., \& Mutschke, H. 1997, A\&A, 327, 743

Houdashelt, M. L., Bell, R. A., Sweigart, A. V., \& Wing, R. F. 2000, AJ, 119, 1424

Ivezić, Ž., \& Elitzur, M. 1995, ApJ, 445, 415

Ivezić, Ž., Nenkova, M., \& Elitzur, M. 1999, User manual for DUSTY. University of Kentucky Internal Report

Jura, M. 1986, ApJ, 303, 327

Jura, M. 1987, ApJ, 313, 743

Jura, M., \& Kleinmann, S. G. 1989, ApJ, 341, 359

Jura, M., \& Kleinmann, S. G. 1990, ApJS, 73, 769

Jura, M., \& Kleinmann, S. G. 1992, ApJS, 79, 105 
Keenan, P. C., \& Boeshaar, P. C. 1980, ApJS, 43, 379

Knapp, G. R., Pourbaix, D., Platais, I., \& Jorissen, A. 2003, A\&A, 403, 993

Lamers, H. J. G. L. M., Zickgraf, F.-J., de Winter, D., Houziaux, L., \& Zorec, J. 1998, A\&A, 340, 117

Lloyd Evans, T. 1983, MNRAS, 204, 985

Loup, C., Zijlstra, A. A., Waters, L. B. F. M., \& Groenewegen, M. A. T. 1997, A\&AS, 125, 419

Marigo, P. 2002, A\&A, 387, 507

Marigo, P., Girardi, L., \& Chiosi, C. 2003, A\&A, 403, 225

Marshall, J. R., van Loon, J. Th., Matsuura, M., et al. 2004, MNRAS, 355,1348

Massey, P., \& Olsen, K. A. G. 2003, AJ, 126, 2867

Mathis, J. S., Rumpl, W., \& Nordsieck, K. H. 1977, ApJ, 217, 425

Matsuura, M., Zijlstra, A. A., van Loon, J. Th., et al. 2002, ApJ, 580, L133

Matsuura, M., Zijlstra, A. A., van Loon, J. Th., et al. 2005, A\&A, submitted

Pégourié, B. 1988, A\&A, 194, 335

Reimers, D. 1975a, in Problems in Stellar Atmospheres and Envelopes, ed. B. Baschek, W. H. Kegel, \& G. Traving (Berlin: Springer), 229

Reimers, D. 1975b, Mem. Soc. R. Sci. Liège 6 Sér., 8, 369

Sandin, C., \& Höfner, S. 2003, A\&A, 398, 253

Schröder, K.-P., Winters, J. M., \& Sedlmayr, E. 1999, A\&A, 349, 898

Trams, N. R., van Loon, J. Th., Waters, L. B. F. M., et al. 1999, A\&A, 346,843
Turnshek, D. E., Turnshek, D. A., Craine, E. R., \& Boeshaar, P. C. 1985, in An Atlas of Digital Spectra of Cool Stars

van Loon, J. Th. 2000, A\&A, 354, 125

van Loon, J. Th., Zijlstra, A. A., Whitelock, P. A., et al. 1997, A\&A, 325,585

van Loon, J. Th., Zijlstra, A. A., Whitelock, P. A., et al. 1998, A\&A, 329, 169

van Loon, J. Th., Zijlstra, A. A., \& Groenewegen, M. A. T. 1999a, A\&A, 346, 805

van Loon, J. Th., Groenewegen, M. A. T., de Koter, A., et al. 1999b, A\&A, 351, 559

van Loon, J. Th., Zijlstra, A. A., Kaper, L., et al. 2001a, A\&A, 368, 239

van Loon, J. Th., Zijlstra, A. A., Bujarrabal, V., \& Nyman, L.-Å. 2001b, A\&A, 368, 950

Wachter, A., Schröder, K.-P., Winters, J. M., Arndt, T. U., \& Sedlmayr, E. 2002, A\&A, 384, 452

Wallerstein, G., Gilroy, K. K., Zethson, T., Johansson, S., \& Hamann, F. 2001, PASP, 113,1210

Whitelock, P. A., Feast, M. W., van Loon, J. Th., \& Zijlstra, A. A. 2003, MNRAS, 342, 86

Wood, P. R., Bessell, M. S., \& Fox, M. W. 1983, ApJ, 272, 99

Wood, P. R., Whiteoak, J. B., Hughes, S. M. G., et al. 1992, ApJ, 397, 552

Zijlstra, A. A., Loup, C., Waters, L. B. F. M., et al. 1996, MNRAS, 279,32 